Prepared FOR SUBMission TO JHEP

\title{
On Consistent Theories of Massive Spin-2 Fields Coupled to Gravity
}

\author{
S.F. Hassan, Angnis Schmidt-May and Mikael von Strauss \\ Department of Physics $\&$ The Oskar Klein Centre, \\ Stockholm University, AlbaNova University Centre, SE-106 91 Stockholm, Sweden \\ E-mail: fawad@fysik.su.se, angnis.schmidt-may@fysik.su.se, \\ mvs@fysik.su.se
}

ABstract: We consider the issues that arise out of interpreting the ghost-free bimetric theory as a theory of a spin-2 field coupled to gravity. This requires identifying a gravitational metric and parameterizing deviations of the resulting theory from general relativity. To this end, we first consider the most general bimetric backgrounds for which a massless and a massive spin-2 fluctuation exist, and we compute the most general expression for the Fierz-Pauli mass. These backgrounds coincide with solutions in general relativity. Based on this, we obtain nonlinear extensions of the massive and massless spin-2 fields. The background value of the nonlinear massive field parameterizes generic deviations of the bimetric theory from GR. It is also shown that the most natural nonlinear massless field does not have standard ghost-free matter couplings, and hence cannot represent the gravitational metric. However, an appropriate gravitational metric can still be identified in the weak gravity limit. Hence in the presence of other neutral spin-2 fields, the weak gravity limit is crucial for compatibility with general relativity. We also write down the action in terms of the nonlinear massive spin-2 field and obtain its ghost-free couplings to matter. The discussion is then generalized to multimetric theories.

KEYWORDS: modified gravity, massive gravity, higher spin fields

\section{Contents}

1 Introduction, motivation and summary $\quad 1$

1.1 Issues considered and summary of results 2

1.2 Background to bimetric theories 4 
2 Proportional-background solutions in bimetric theory 5

2.1 Review of the ghost-free bimetric theory 6

2.2 Proportional background solutions 7

2.3 Discussion 8

3 Linear massive and massless modes $\quad 9$

3.1 Massive and massless modes in the linearized theory 9

$\begin{array}{lll}3.2 & \text { Weak gravity limit } & 10\end{array}$

4 The nonlinear massless and massive modes $\quad 11$

4.1 The nonlinear massless spin-2 field $G \quad 11$

4.2 The nonlinear massive spin-2 field $M \quad 13$

4.3 Absence of ghost-free matter coupling of the massless mode $G \quad 15$

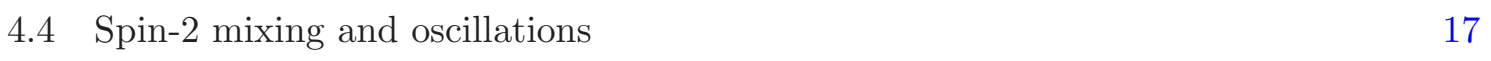

5 Action for the nonlinear massive spin-2 field $\quad 17$

5.1 The action in terms of $g$ and $M$

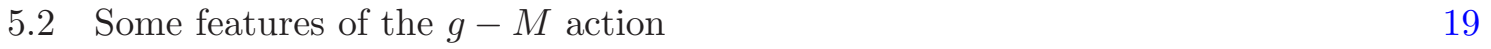

5.3 Coupling massive spin-2 fields to matter 20

6 Generalization to more than one massive field 22

$\begin{array}{lll}7 & \text { Discussion } & 24\end{array}$

$\begin{array}{ll}\text { A Curvature relations } & 25\end{array}$

B Bimetric action in scaled variables $\quad 25$

C Details of the nonlinear $G-M^{G}$ action $\quad 26$

\section{Introduction, motivation and summary}

Theories of interacting spin-2 fields have been considered over many years with various motivations, for example, in [1-6], or more recently in [7-15]. Often, these are formulated in terms of two metrics, $g_{\mu \nu}$ and $f_{\mu \nu}$, with non-derivative interactions. These theories generically contain Boulware-Deser ghost instabilities [16]. The bimetric theories that avoid this problem were written down and proven to be ghost-free in $[17,18]$. This was based on [19-21] that further developed the massive gravity work in [22, 23], as will be briefly reviewed below. 
More recently, this was extended to ghost-free theories of many spin-2 fields in terms of $\mathcal{N}$ vielbeins [24], while a formulation in terms of $\mathcal{N}$ metrics is given in [25]. ${ }^{1}$

In the ghost-free bimetric theory [17], a priori, the two spin-2 fields $g_{\mu \nu}$ and $f_{\mu \nu}$ appear more or less on the same footing. For obvious reasons, eventually we would like to interpret this as a theory of a "massive" spin-2 field interacting with gravity. Furthermore, the gravity sector of the theory should not show observable deviations from tested aspects of general relativity. In this paper we consider the issues that arise when using the bimetric theory to describe a spin-2 field coupled to gravity. The considerations also apply to the multivielbein/multimetric case.

To focus attention, the ghost-free bimetric theory that we will work with has the form,

$$
S=\int \mathrm{d}^{4} x\left[m_{g}^{2} \sqrt{-g} R(g)+m_{f}^{2} \sqrt{-f} R(f)-2 m^{4} \sqrt{-g} V\left(g^{-1} f, \beta_{n}\right)\right]+S_{m}\left(g, f, \psi_{m}\right),
$$

with details to be specified later (2.1), (2.2). The particular combination of kinetic and potential terms renders the theory ghost-free. The seven parameters of the theory are $m_{p}$, $m_{f}$ and five $\beta_{n}$. The simplest possible "matter" interactions that are also known to be ghost-free [17], are of the form,

$$
\sqrt{-g} \mathcal{L}_{g}(g, \psi)+\sqrt{-f} \mathcal{L}_{f}\left(f, \psi^{\prime}\right) .
$$

Other forms of matter coupling should be explicitly checked for ghosts. Generic cosmological and localized solutions in this theory could show large deviations from solutions in general relativity (GR) although there also exist classes of solutions that are close to GR spacetimes [26-32].

Below, we will first describe the issues that arise out of interpreting (1.1) as a theory of a spin-2 field coupled to gravity, and summarize our results. Then we will briefly review the development of spin-2 theories with emphasis on the importance of the nonlinear methods.

\subsection{Issues considered and summary of results}

By construction, in (1.1) around flat backgrounds $\bar{g}_{\mu \nu}=\bar{f}_{\mu \nu}=\eta_{\mu \nu}$ (that exist for a restricted set of $\beta_{n}$ ), the fluctuations $\delta g_{\mu \nu}$ and $\delta f_{\mu \nu}$ are linear combinations of a massless spin-2 mode $\delta G_{\mu \nu}$ (2 polarizations) and a massive spin-2 mode $\delta M_{\mu \nu}$ (5 polarizations) with a Fierz-Pauli mass term $[33,34]$. At nonlinear level too, the theory has 7 propagating degrees of freedom, although in that case the analogue of the decomposition in terms of mass is not known. An obvious problem is to specify the most general class of backgrounds around which the theory has well defined massive and massless fluctuations, and to compute the spectrum as a function of the unrestricted $\beta_{n}$.

To regard (1.1) as a theory of a neutral spin-2 field interacting with gravity, one has to first identify the gravitational metric, say $g^{\mathrm{GR}}$, in terms of $g$ and $f$. An important restriction

\footnotetext{
${ }^{1}$ In hindsight, it turns out that Chamseddine, Salam and Strathdee in 1978 [5] had a ghost-free bimetric theory, written in terms of vielbeins and with supersymmetry, although the absence of the BD ghost could not be demonstrated then.
} 
is that the standard minimal couplings of $g^{\mathrm{GR}}$ to matter, demanded by the weak equivalence principle, should also be ghost-free. A first guess for $g^{\mathrm{GR}}$, one suggested as far back as [2], is the nonlinear extension of the massless mode $\delta G_{\mu \nu}$. But to explicitly check if this allows for ghost-free matter couplings, one needs an explicit nonlinear expression for it in terms of $g$ and $f$. The other obvious fall back options are $g$ or $f$. While not mass eigenstates, these have ghost-free matter couplings.

Having identified a gravitational metric, the next task is to verify that the theory has parameter space regions where the solutions for $g^{\text {GR }}$ are close enough to GR solutions that the bimetric theory is not immediately ruled out on observational grounds. For this, if possible, one would like to have some criteria or quantity to parameterize deviations of the bimetric theory from GR. In this paper we consider these issues and the results are summarized below.

Proportional backgrounds and general mass eigenstates: To obtain the mass spectrum, we consider the most general class of bimetric backgrounds around which a massive mode with a well defined Fierz-Pauli mass term exists. These are the proportional backgrounds $\bar{f}_{\mu \nu}=c^{2} \bar{g}_{\mu \nu}$, where $c$ is determined by the parameters of the theory. They coincide exactly with solutions in general relativity with a cosmological constant, and always exist as bimetric vacuum solutions without fixing the parameters of the theory, as long as real solutions for $c$ exist. Flat space solutions require fixing one of the seven parameters by setting the cosmological constant to zero. The solutions also exist in the presence of sources, as long as the sources of the $g$ and $f$ equations of motion satisfy $m_{g}^{2} \bar{T}_{\mu \nu}^{f}=m_{f}^{2} \bar{T}_{\mu \nu}^{g}$. This constraint is not natural, but shows that deviations from it drive bimetric solutions away from GR solutions in a generic sense (although it is still possible to get isolated GR type solutions).

Considering fluctuations around $\bar{f}_{\mu \nu}=c^{2} \bar{g}_{\mu \nu}$ backgrounds, we obtain the most general expression for the Fierz-Pauli mass, as well as the expressions for the massless mode $\delta G_{\mu \nu}$ and the massive mode $\delta M_{\mu \nu}$.

Nonlinear modes: We give a procedure to systematically obtain nonlinear combinations of $f$ and $g$ that reduce to $\delta G$ and $\delta M$ at the linear level. Although there are infinitely many such combinations we identify one, $G_{\mu \nu}$, as the nonlinear extension of the massless mode and two possible candidates, $M_{\mu \nu}$ and $M_{\mu \nu}^{G}$, for the nonlinear extension of the massive mode, based on reasonable criteria. These seem natural and are simple enough that the expressions relating them to $g$ and $f$ are invertible. The vanishing of the nonlinear massive mode, $M=0$, is in one-to-one correspondence with occurrence of proportional backgrounds $\bar{f}_{\mu \nu}=c^{2} \bar{g}_{\mu \nu}$. Hence deviations of the VEV of $M$ from 0 are driven by the matter couplings of the spin-2 fields and parameterize generic deviations of the bimetric theory from GR. If these nonlinear modes have a relevance directly at the nonlinear level is not yet answered.

Identification of gravity: Having a nonlinear massless mode $G$ in hand, we can test the conjecture that it should be identified as the gravitational metric. Through an ADM analysis we show that within the bimetric framework, the standard minimal coupling of $G_{\mu \nu}$ to matter is not ghost-free. This rules out that particular $G_{\mu \nu}$ as a candidate for the gravitational metric. 
Another option is $g_{\mu \nu}$ (or equivalently $f_{\mu \nu}$ since the formulation is symmetric) which has a ghost-free matter coupling. In particular, in the limit $m_{g}>c m_{f}$, we have $\delta G \rightarrow \delta g$ and $\delta M \rightarrow \delta f$. For the nonlinear fields too, in the limit, $m_{g}>>m_{f}, G \rightarrow g$, although in this case $M$ has no particular limit. Hence, if one identifies $g_{\mu \nu}$ as the gravitational metric, then in the weak gravity limit, $g_{\mu \nu}$ will mostly consist of the massless mode. An obvious consequence is that in the presence of massive spin-2 fields, metric perturbations created by a matter source will also have a small massive component.

Now one can regard the pair $g, M$ as the basic variables and express the bimetric action in terms of them. Although the kinetic part in terms of $M$ is more involved than the original form in terms of $f$, the potential is now a finite polynomial in $M$ and does not involve a square-root matrix. Also as pointed out earlier, couplings that drive $M$ away from $\bar{M}=0$, also drive the solutions for the metric $g$ away from GR. Subsequently, from the ghost-free couplings of $f_{\mu \nu}$ to matter, we obtain couplings between the massive field $M$ and matter.

Multi spin-2 fields coupled to gravity: Finally we extend the above considerations to multi spin-2 theories, as theories of $\mathcal{N}-1$ massive spin-2 fields coupled to gravity.

\subsection{Background to bimetric theories}

The BD ghost was first observed in massive gravity [16] which corresponds to the bimetric theory with one metric held fixed, say, $f_{\mu \nu}=\eta_{\mu \nu}$. It led to the speculation that such ghostfree theories may not exist. The major breakthrough came with the work of de Rham, Gabadadze and Tolley [22, 23], who obtained a potentially ghost-free nonlinear massive gravity action for $f_{\mu \nu}=\eta_{\mu \nu}$, the dRGT model, on which subsequent developments are based. The construction was based on a "decoupling limit" analysis (developed for the purpose [12, 35]) which guaranteed the absence of ghost in that limit. In the perturbative approach, it becomes difficult to extend the analysis beyond the decoupling limit, although [23] outlined an argument to show the absence of ghost in the Hamiltonian [36] formalism to quartic order in $h_{\mu \nu}=g_{\mu \nu}-\eta_{\mu \nu}$.

To proceed any further, one had to first insure that the BD ghost is indeed absent in the dRGT model for a nonlinear $g_{\mu \nu}$. One also needs to find out if the more natural case of massive gravity with a non-flat reference metric, $f_{\mu \nu} \neq \eta_{\mu \nu}$, is ghost-free. It turns out that the "decoupling limit", wielded powerfully in $[22,35]$, is not adequate to address these situations, despite some claims to the contrary in [37] and some of its citations. ${ }^{2}$

\footnotetext{
${ }^{2}$ The decoupling limit analysis does not extend beyond the decoupling limit for two obvious reasons: (1) It involves working with Stückelberg fields $\phi^{a}$, introduced via $f_{\mu \nu}=\partial_{\mu} \phi^{a} \partial_{\nu} \phi^{b} \eta_{a b}$, rather than with the metric $g_{\mu \nu}$. The $\phi^{a}$ mix only with the 4 "gauge" modes of $g_{\mu \nu}$ under coordinate transformations and learn about the potential $\mathrm{BD}$ ghost through them. But the BD ghost mostly resides in the remaining 6 components of the metric and cannot be completely transferred to the Stückelberg fields by coordinate transformations (otherwise, ghost fluctuations would be expressible as $\nabla_{(\mu} \xi_{\nu)}$ and would not contribute to interactions between conserved sources). Similarly, it is incorrect to argue that the number of independent modes in $g_{\mu \nu}$ can be reduced to 2 simply by coordinate transformations, by citing the analogy with GR. In GR this counting is done on-shell and holds only for the solutions of the massless Einstein's equations. Such a counting does not hold for the massive gravity equations. It has also been argued that one can transform $g_{\mu \nu}$ to $\eta_{\mu \nu}$ by a coordinate transformation
} 
The nonlinear analysis that can answer the above questions was developed in $[20,21]$ based on the formalism of [19]. In [20], for the case $f_{\mu \nu}=\eta_{\mu \nu}$, it was proven that the dRGT model was ghost-free at the nonlinear level. This conclusively established the absence of the $\mathrm{BD}$ ghost for the first time. The theory with generic non-flat $f_{\mu \nu}$ was considered first in [21] and also proven to be ghost-free nonlinearly. This generic $f_{\mu \nu}$ theory provides the most natural setup for discussing massive gravity. But from the point of view of this paper, it describes rather a massive spin-2 field $g_{\mu \nu}$ in a non-dynamical gravitational background $f_{\mu \nu}$.

Finally, [17] obtained the ghost-free bimetric theory for two interacting spin-2 fields $g_{\mu \nu}$ and $f_{\mu \nu}$ with the correct kinetic structure, by exploiting the symmetries of the interactions. An issue raised in [39] about the existence of a secondary constraint that was needed for the consistency of the formalism was cleared up in [18]. In this paper we will work mostly with this Hassan-Rosen bimetric theory. For related work, see [40-52].

The rest of the paper is organized as follows. In section 2, we review the ghost-free bimetric theory and discuss the proportional background solutions. In section 3, we obtain the linear mass eigenstates and compute the general expression for the FP mass. We also discuss the weak gravity limit. In section 4, we obtain the nonlinear massless and massive spin-2 modes and show that the massless mode does not have ghost-free minimal matter couplings. In section 5, we express the bimetric action in terms of $g$ and the massive mode $M$ and discuss some of its features. We also discuss the coupling of the massive spin-2 field $M$ to matter. In section 6 , the discussion is extended to multimetric theories. Section 7 contains a brief discussion of the results and some comments. Appendix A summarizes some useful equations used in the text. Appendix B describes a rescaling that render the action more symmetric. Finally appendix $\mathrm{C}$ contains the details of the bimetric action in terms of the nonlinear massless and massive modes $G$ and $M^{G}$.

\section{Proportional-background solutions in bimetric theory}

Generic solutions of the bimetric theory have little resemblance to solutions in general relativity. In this section we concentrate on a particular class of bimetric background solutions that are indistinguishable from backgrounds in general relativity. Although very restrictive, this helps in identifying bimetric theories that are close to general relativity. The solutions are also useful in analyzing the linear and nonlinear mass spectrum of the bimetric theory. We begin with a review of the ghost-free bimetric action.

and then apply the decoupling limit. This argument ignores the elementary fact that one cannot choose a locally flat coordinate systems over the entire spacetime. Obviously, the flat space action and equations of motion are not the same as the curved space ones. Thus, away from the decoupling limit it is not enough to study the $\phi^{a}$ alone, ignoring $g_{\mu \nu}$ and the potential ghost within it, by invoking the above arguments. (2) So far, it is not obvious how to obtain a decoupling limit for a generic non-flat $f_{\mu \nu}$. For example, see [38] for a recent attempt to find a decoupling limit for a de Sitter $f_{\mu \nu}$. 


\subsection{Review of the ghost-free bimetric theory}

Here we briefly review the ghost-free bimetric action and equations of motion. The ghost-free bimetric action, excluding matter couplings, is [17],

$$
S_{g f}=\int \mathrm{d}^{4} x\left[m_{g}^{2} \sqrt{-\operatorname{det} g} R_{g}+m_{f}^{2} \sqrt{-\operatorname{det} f} R_{f}-2 m^{4} \sqrt{-\operatorname{det} g} V\left(\sqrt{g^{-1} f} ; \beta_{n}\right)\right] .
$$

The potential $V$ is given by,

$$
V\left(\mathbb{X} ; \beta_{n}\right)=\sum_{n=0}^{4} \beta_{n} e_{n}(\mathbb{X})
$$

where, $e_{n}(\mathbb{K})$ are elementary symmetric polynomials of the eigenvalues of the matrix $\mathbb{X}$. In 4 dimensions they can be expressed as,

$$
e_{0}=1, \quad e_{1}=[\mathbb{X}], \quad e_{2}=\frac{1}{2}\left([\mathbf{X}]^{2}-\left[\mathbb{K}^{2}\right]\right), \quad e_{3}=\frac{1}{6}\left([\mathbf{X}]^{3}-3[\mathbf{X}]\left[\mathbb{K}^{2}\right]+2\left[\mathbb{K}^{3}\right]\right), \quad e_{4}=\operatorname{det}(\mathbb{X}),
$$

where, [ ] denotes the matrix trace. This potential $V$ was first suggested, for $f_{\mu \nu}=\eta_{\mu \nu}$ in $[22,23]$ as the unique candidate for a ghost-free massive gravity, based on a "decoupling limit" analysis. That it was ghost-free nonlinearly was proven in [20]. The theories with general and dynamical $f_{\mu \nu}$ where first considered and shown to be ghost-free in [17-19, 21]. The square root matrix $\mathbb{X}=\sqrt{g^{-1} f}$ in $V$ is necessary to avoid the ghost, but also complicates the analysis.

The independent parameters in the action (2.1) are the five dimensionless $\beta_{n}$ and the two "Planck masses", $m_{g}$ and $m_{f}$. The mass scale $m$ is degenerate with the $\beta_{n}$ and can be expressed in terms of the other mass parameters. Integrating out matter fields coupled to the $g$ and $f$ metrics respectively, results in vacuum energy contributions to $\beta_{0}$ and $\beta_{4}$. The remaining $\beta_{n}$ measure the strength of nonlinear interactions between the two metrics. An important property of $V$ is,

$$
\sqrt{-\operatorname{det} g} V\left(\sqrt{g^{-1} f} ; \beta_{n}\right)=\sqrt{-\operatorname{det} f} V\left(\sqrt{f^{-1} g} ; \beta_{4-n}\right) .
$$

Then, the action (2.1) is symmetric under the simultaneous replacements,

$$
g \leftrightarrow f, \quad \beta_{n} \rightarrow \beta_{4-n}, \quad m_{g} \leftrightarrow m_{f} .
$$

The $g_{\mu \nu}$ and $f_{\mu \nu}$ equations of motion with generic "matter" couplings are [19],

$$
\begin{aligned}
& R_{\mu \nu}(g)-\frac{1}{2} g_{\mu \nu} R(g)+\frac{m^{4}}{m_{g}^{2}} V_{\mu \nu}^{g}=\frac{1}{m_{g}^{2}} T_{\mu \nu}^{g}, \\
& R_{\mu \nu}(f)-\frac{1}{2} f_{\mu \nu} R(f)+\frac{m^{4}}{m_{f}^{2}} V_{\mu \nu}^{f}=\frac{1}{m_{f}^{2}} T_{\mu \nu}^{f} .
\end{aligned}
$$

Here, the stress-energy tensors are defined by $T_{\mu \nu}^{g}=-(1 / \sqrt{g}) \delta S_{m} / \delta g^{\mu \nu}$, and similarly for $T_{\mu \nu}^{f}$, where $S_{m}$ is the matter action added to $S_{g f}$ as in (1.1). The interaction contributions $V_{\mu \nu}^{g}$ and $V_{\mu \nu}^{f}$ are explicitly given by,

$$
V_{\mu \nu}^{g}=\sum_{n=0}^{3}(-1)^{n} \beta_{n} g_{\mu \lambda} Y_{(n) \nu}^{\lambda}\left(\sqrt{g^{-1} f}\right), \quad V_{\mu \nu}^{f}=\sum_{n=0}^{3}(-1)^{n} \beta_{4-n} f_{\mu \lambda} Y_{(n) \nu}^{\lambda}\left(\sqrt{f^{-1} g}\right)
$$


where the matrices $Y_{(n) \nu}^{\mu}(\mathbb{X})$ can be expressed as,

$$
Y_{(n)}(\mathbb{X})=\sum_{r=0}^{n}(-1)^{r} \mathbb{X}^{n-r} e_{r}(\mathbb{X}) .
$$

The two expressions in (2.8) are related through the replacements (2.5). In this sense, the bimetric theory treats $g_{\mu \nu}$ and $f_{\mu \nu}$ on the same footing.

The usual Bianchi identities of the curvature tensors together with $\nabla_{g, f}^{\mu} T_{\mu \nu}^{g, f}=0$, imply the Bianchi constraints, which are independent of the scales $m_{g}$ and $m_{f}$,

$$
\nabla_{g}^{\mu} V_{\mu \nu}^{g}=0, \quad \nabla_{f}^{\mu} V_{\mu \nu}^{f}=0 .
$$

\subsection{Proportional background solutions}

Generic solutions of the bimetric theory are very different from solutions in general relativity. Here we consider a particular class of bimetric solutions $\bar{g}_{\mu \nu}$ and $\bar{f}_{\mu \nu}$, sourced by $\bar{T}_{\mu \nu}^{g}$ and $\bar{T}_{\mu \nu}^{f}$, which coincide with solutions for the metric in GR. These are solutions of the type ${ }^{3}$

$$
\bar{f}_{\mu \nu}=c^{2} \bar{g}_{\mu \nu},
$$

and exist only if $\bar{T}_{\mu \nu}^{f} \propto \bar{T}_{\mu \nu}^{g}$. This restriction on the matter sources is not always realistic, but such solutions are motivated by other considerations discussed at the end of this section.

For the ansatz (2.11), the Bianchi constraints (2.10) imply that $c$ is a constant. Then (2.6) and (2.7) reduce to two copies of Einstein's equations for the curvatures of $\bar{g}_{\mu \nu}$,

$$
\bar{R}_{\mu \nu}-\frac{1}{2} \bar{g}_{\mu \nu} \bar{R}+\Lambda_{g} \bar{g}_{\mu \nu}=\frac{1}{m_{g}^{2}} \bar{T}_{\mu \nu}^{g}, \quad \bar{R}_{\mu \nu}-\frac{1}{2} \bar{g}_{\mu \nu} \bar{R}+\Lambda_{f} \bar{g}_{\mu \nu}=\frac{1}{m_{f}^{2}} \bar{T}_{\mu \nu}^{f},
$$

where the cosmological constants are given by,

$$
\Lambda_{g}=\frac{m^{4}}{m_{g}^{2}}\left(\beta_{0}+3 c \beta_{1}+3 c^{2} \beta_{2}+c^{3} \beta_{3}\right), \quad \Lambda_{f}=\frac{m^{4}}{m_{f}^{2} c^{2}}\left(c \beta_{1}+3 c^{2} \beta_{2}+3 c^{3} \beta_{3}+c^{4} \beta_{4}\right) .
$$

Obviously, the equations are consistent only if,

$$
\left(\Lambda_{g}-\Lambda_{f}\right) \bar{g}_{\mu \nu}=\left(m_{g}^{-2} \bar{T}_{\mu \nu}^{g}-m_{f}^{-2} \bar{T}_{\mu \nu}^{f}\right) .
$$

The vacuum energy contributions to $\bar{T}_{\mu \nu}^{g}$ and $\bar{T}_{\mu \nu}^{f}$ can always be absorbed in $\beta_{0}$ and $\beta_{4}$. Hence, the right-hand side can be assumed to contain no piece proportional to $\bar{g}_{\mu \nu}$. Then, for localizable sources, each side of the above equation must vanish separately, ${ }^{4}$

$$
\Lambda_{g}=\Lambda_{f}, \quad \bar{T}_{\mu \nu}^{f}=\frac{m_{f}^{2}}{m_{g}^{2}} \bar{T}_{\mu \nu}^{g} .
$$

Later it will be seen that (2.15) is also crucial for the existence of spin-2 massive and massless eigenstates.

\footnotetext{
${ }^{3}$ For non-proportional metrics, GR type solutions exist for certain choices of $\beta_{n}$, but for specific metric ansatz, say the FRW ansatz [28, 29].

${ }^{4}$ If $\Lambda_{g} \neq \Lambda_{f}$, then (2.12) and (2.14) would lead to a complicated differential equation for the $T_{\mu \nu}$ 's.
} 
The equation $\Lambda_{g}=\Lambda_{f}$ determines the constant $c$ in terms of the parameters of the theory, $m_{g}, m_{f}$ and the five $\beta_{n}$, through the quartic equation,

$$
\alpha^{2} \beta_{3} c^{4}+\left(3 \alpha^{2} \beta_{2}-\beta_{4}\right) c^{3}+3\left(\alpha^{2} \beta_{1}-\beta_{3}\right) c^{2}+\left(\alpha^{2} \beta_{0}-3 \beta_{2}\right) c-\beta_{1}=0
$$

where

$$
\alpha=\frac{m_{f}}{m_{g}} .
$$

On solving the above equation for $c$ and substituting in $\Lambda_{g}$, one obtains the cosmological constant in terms of the parameters of the theory.

To get a feeling for the behaviour of $c$ we can solve this equation for the simple case of the "minimal" bimetric model, corresponding to $\beta_{1}=\beta_{3}=0$. Then (2.16) gives,

$$
c^{2}=\frac{3 \beta_{2}-\alpha^{2} \beta_{0}}{3 \alpha^{2} \beta_{2}-\beta_{4}}, \quad\left(\beta_{1}=\beta_{3}=0\right),
$$

showing that, in general, $c^{2}$ could have any value depending on $\beta_{0}, \beta_{2}$ and $\beta_{4}$. This in turn gives the cosmological constant,

$$
\Lambda_{g}=\Lambda_{f}=\frac{m^{4}}{m_{g}^{2}} \frac{9 \beta_{2}^{2}-\beta_{0} \beta_{4}}{3 \alpha^{2} \beta_{2}-\beta_{4}}, \quad\left(\beta_{1}=\beta_{3}=0\right) .
$$

The scale $m^{4}$ can be eliminated in terms of the Fierz-Pauli mass of the massive excitation given in the next subsection.

Note that the most general set of parameters for which the theory admits flat space as a solution is obtained from $\Lambda_{g}=0$, after solving for $c$. This condition can be solved for one of the $\beta_{n}$, leaving the rest free. In contrast, specifying flat space through $\bar{f}=\bar{g}=\eta$ and $\Lambda_{g}=\Lambda_{f}=0$ will eliminate two of the $\beta_{n}$ leading to a smaller parameter space. For example, in the minimal case considered above, theories that admit flat space as a background are parameterized by $9 \beta_{3}^{2}=\beta_{0} \beta_{4}$, whereas forcing $c=1$ gives the smaller parameter space $\beta_{0}=\beta_{4}=-3 \beta_{3}$.

\subsection{Discussion}

If one interprets one of the two metrics, say $\bar{g}_{\mu \nu}$, as the gravitational metric coupled to ordinary matter $\bar{T}^{g}$ with Planck mass $m_{g}$, then one recovers all classical backgrounds of GR. However, the requirement $\bar{T}_{\mu \nu}^{f}=\alpha^{2} \bar{T}_{\mu \nu}^{g}$ imposed by these solutions is not realistic (except possibly for $\alpha=1$ such that the two metrics are coupled to the same matter). In spite of this such backgrounds are motivated by other considerations.

(1) The ansatz (2.11) results in the most general class of bimetric backgrounds for which there exist a well-defined massive spin-2 fluctuation $\delta M_{\mu \nu}$ with a Fierz-Pauli structure,

$$
m_{\mathrm{FP}} \sqrt{-\operatorname{det} \bar{g}}\left[\delta M_{\nu}^{\mu} \delta M_{\mu}^{\nu}-\left(\delta M_{\mu}^{\mu}\right)^{2}\right]
$$

along with a decoupled massless spin-2 fluctuation $\delta G_{\mu \nu}$, as will be discussed below. The explicit expressions help extend the linear mass eigenstates $\delta M$ and $\delta G$ to nonlinear fields $M$ 
and $G$. The $c \neq 1$ case helps in identifying $G$. Finally, we interpret the bimetric theory as a nonlinear theory of a massive spin-2 field coupled to gravity.

(2) To be consistent with observations, the solutions for the nonlinear field that is identified with the gravitational metric must be very close to the corresponding solutions in GR. More specifically, corrections to the GR solutions coming from the non-gravitational sector must be strongly suppressed in the weak gravity limit. These issues are difficult to investigate nonlinearly. Thus as a first step, one can consider perturbations around backgrounds of the type (2.11), sourced by independent $\delta T^{g}$ and $\delta T^{f}$ to probe parameter regions that suppress deviations from GR. Later we will also see that the nonlinear massive field defined with respect to these backgrounds probes deviations from GR.

\section{Linear massive and massless modes}

In Minkowski backgrounds $\bar{g}=\bar{f}=\eta$, where the concept of mass is well defined through the Poincaré group, the spectrum of bimetric theory is known to consist of a massive and a massless spin-2 fluctuation $[2,17]$. Such backgrounds exist only after two out of the five $\beta_{n}$ parameters are fixed. Here we consider the spectrum of linear fluctuations in the theory with arbitrary $\beta_{n}$. In non-flat backgrounds we define a massive fluctuation as one with a FierzPauli mass term (2.20). In bimetric theory, such mass terms arise only around proportional

backgrounds $\bar{f}_{\mu \nu}=c^{2} \bar{g}_{\mu \nu}$ considered above. For independent source fluctuations $\delta T_{\mu \nu}^{g}$ and $\delta T_{\mu \nu}^{f}$ the expressions help in characterizing deviations from GR. The linear mass eigenstates are extended to nonlinear fields in the next section.

\subsection{Massive and massless modes in the linearized theory}

Consider canonically normalized fluctuations around the $\bar{f}_{\mu \nu}=c^{2} \bar{g}_{\mu \nu}$ backgrounds,

$$
g_{\mu \nu}=\bar{g}_{\mu \nu}+\frac{1}{m_{g}} \delta g_{\mu \nu}, \quad f_{\mu \nu}=c^{2} \bar{g}_{\mu \nu}+\frac{c}{m_{f}} \delta f_{\mu \nu} .
$$

Then to linear order,

$$
\left(\sqrt{g^{-1} f}\right)_{\nu}^{\rho}=c \delta_{\nu}^{\rho}+\delta S_{\nu}^{\rho}, \quad \text { where, } \quad \delta S^{\rho}{ }_{\nu}=\frac{1}{2 m_{f}} \bar{g}^{\rho \mu}\left(\delta f_{\mu \nu}-c \frac{m_{f}}{m_{g}} \delta g_{\mu \nu}\right) .
$$

Expanding the interaction contributions (2.8) and using the results in appendix (A) gives the linearized equations,

$$
\begin{aligned}
& \overline{\mathcal{E}}_{\mu \nu}^{\rho \sigma} \delta g_{\rho \sigma}+\Lambda_{g} \delta g_{\mu \nu}-\frac{m^{4} B}{m_{g}} \bar{g}_{\mu \rho}\left(\delta S^{\rho}{ }_{\nu}-\delta_{\nu}^{\rho} \delta S^{\sigma}{ }_{\sigma}\right)=\frac{1}{m_{g}} \delta T_{\mu \nu}^{g}, \\
& \overline{\mathcal{E}}_{\mu \nu}^{\rho \sigma} \delta f_{\rho \sigma}+\Lambda_{f} \delta f_{\mu \nu}+\frac{m^{4} B}{c m_{f}} \bar{g}_{\mu \rho}\left(\delta S^{\rho}{ }_{\nu}-\delta_{\nu}^{\rho} \delta S^{\sigma}{ }_{\sigma}\right)=\frac{1}{m_{f}} \delta T_{\mu \nu}^{f},
\end{aligned}
$$

where,

$$
B=\frac{1}{c}\left(c \beta_{1}+2 c^{2} \beta_{2}+c^{3} \beta_{3}\right) .
$$

$\overline{\mathcal{E}}$ is given in (A.7). By taking appropriate linear combinations, (3.3) and (3.4) can be easily decoupled in terms of a massive $\left(\delta M_{\mu \nu} \sim \bar{g}_{\mu \lambda} \delta S_{\nu}^{\lambda}\right)$ and a massless $\delta G_{\mu \nu} \sim \delta g_{\mu \nu}+c\left(m_{f} / m_{g}\right) \delta f_{\mu \nu}$ 
spin-2 fluctuation. However, this is possible only if $\Lambda_{g}=\Lambda_{f}$, which was also required on other grounds. Finally, the canonically normalized massless and massive fluctuations become ${ }^{5}$

$$
\begin{aligned}
\delta G_{\mu \nu} & =\frac{1}{\sqrt{c^{2} \alpha^{2}+1}}\left(\delta g_{\mu \nu}+c \alpha \delta f_{\mu \nu}\right), \\
\delta M_{\mu \nu} & =\frac{1}{\sqrt{c^{2} \alpha^{2}+1}}\left(\delta f_{\mu \nu}-c \alpha \delta g_{\mu \nu}\right),
\end{aligned}
$$

where $\alpha=\frac{m_{f}}{m_{g}}$. The corresponding massless and massive equations are,

$$
\begin{aligned}
& \overline{\mathcal{E}}_{\mu \nu}^{\rho \sigma} \delta G_{\rho \sigma}+\Lambda_{g} \delta G_{\mu \nu}=\frac{\delta T_{\mu \nu}^{(g)}+c^{2} \delta T_{\mu \nu}^{(f)}}{m_{g} \sqrt{c^{2} \alpha^{2}+1}} \\
& \overline{\mathcal{E}}_{\mu \nu}^{\rho \sigma} \delta M_{\rho \sigma}+\Lambda_{g} \delta M_{\mu \nu}+\frac{m_{\mathrm{FP}}^{2}}{2}\left(\delta M_{\mu \nu}-\bar{g}_{\mu \nu} \bar{g}^{\rho \sigma} \delta M_{\rho \sigma}\right)=c \frac{\delta T_{\mu \nu}^{(f)}-\alpha^{2} \delta T_{\mu \nu}^{(g)}}{m_{f} \sqrt{c^{2} \alpha^{2}+1}}
\end{aligned}
$$

The Fierz-Pauli mass above is parameterized as,

$$
m_{\mathrm{FP}}^{2}=m^{4}\left(c \beta_{1}+2 c^{2} \beta_{2}+c^{3} \beta_{3}\right)\left(\frac{1}{c^{2} m_{f}^{2}}+\frac{1}{m_{g}^{2}}\right) .
$$

From (3.8) it is evident that, in the background metric $\bar{g}_{\mu \nu}$, the massless fluctuation $\delta G_{\mu \nu}$ couples to matter with the effective Planck mass,

$$
m_{p}=m_{g} \sqrt{c^{2} \alpha^{2}+1}=\sqrt{m_{g}^{2}+c^{2} m_{f}^{2}} .
$$

which must be large for gravity to be weak. This can be achieved in different ways with different consequences. It is also evident that at the linear level, $\delta G$ behaves like the metric perturbation in GR. Deviations from GR emerge mainly at the nonlinear level.

Away from proportional backgrounds, the fluctuations generically do not have a FierzPauli mass term. The analysis is further complicated by the fact that in such cases, $\sqrt{g^{-1} f}$ does not have a simple expansion.

\subsection{Weak gravity limit}

In GR, gravity is described in terms of a massless spin-2 field minimally coupled to matter, as required by the weak equivalence principle. Considering the observational evidence in support of GR, it is natural to assume that in interacting spin-2 theories too, the gravitational interactions must be associated predominantly, if not exclusively, with the massless spin-2 mode of the theory. The validity of the weak equivalence principle then requires that this gravitational mode must couple to matter in more or less the same way that the gravitational metric couples to matter in GR. This simple observation leads to the following possibilities.

(1) Let's assume that the massless mode $\delta G_{\mu \nu}$ can be extended to a nonlinear field $G_{\mu \nu}$. If $G_{\mu \nu}$ could directly couple to matter in a ghost-free manner, using the same minimal

\footnotetext{
${ }^{5}$ The canonical normalization is determined from the action requiring that $\delta g \overline{\mathcal{E}} \delta g+\delta f \overline{\mathcal{E}} \delta f=\delta M \overline{\mathcal{E}} \delta M+$ $\delta G \overline{\mathcal{E}} \delta G$. This value will change if $\overline{\mathcal{E}}$ on the rhs is computed with the background metric $\bar{G}$ instead of $\bar{g}$.
} 
coupling prescription as in GR, then such matter couplings would not directly violate the weak equivalence principle. In this case, one should express the operator $\overline{\mathcal{E}}_{\mu \nu}^{\rho \sigma}$ in (3.8) in terms of the background $\bar{G}_{\mu \nu}$ which will be proportional to $\bar{g}_{\mu \nu}$, giving, $\overline{\mathcal{E}}_{\mu \nu}^{\rho \sigma}(\bar{g})=a\left(c, m_{f}, m_{g}\right) \overline{\mathcal{E}}_{\mu \nu}^{\rho \sigma}(\bar{G})$. Then the Planck mass is $a m_{p}$ which must be large. Later we identify a nonlinear massless mode $G_{\mu \nu}$ and show that it cannot couple to matter in a ghost-free way. In the absence of consistent direct couplings of $G_{\mu \nu}$ to matter, a different approach is needed.

(2) Now consider setups where matter fields can directly couple only to the metrics $g_{\mu \nu}$ or $f_{\mu \nu}$ (as in (1.2)), but not to $G_{\mu \nu}$. This would be a natural way of accommodating the weak equivalence principle only if $g_{\mu \nu}$ or $f_{\mu \nu}$ described gravity. On the other hand, empirically, gravity is well described by a massless spin-2 field, which in the bimetric setup is $G_{\mu \nu}$. These two requirements can be reconciled if the massless mode $G_{\mu \nu}$ is dominated by $g_{\mu \nu}$ or $f_{\mu \nu}$. Here we consider the possibility that $G_{\mu \nu}$ is mostly made up of $g_{\mu \nu} .{ }^{6}$ The limits in which this holds can be identified at the linearized level from (3.6), where, $\delta G_{\mu \nu} \sim \delta g_{\mu \nu}$ holds in the limit

$$
m_{g}>>c m_{f} .
$$

This can be achieved by a small $m_{f}$ or a small $c$ of both. Whether this choice is natural or not, will not be addressed here. Also in this limit, the massive fluctuation $\delta M_{\mu \nu}$ is mostly saturated by $f_{\mu \nu}$. The strength of $\delta M_{\mu \nu}$ interactions depend on the relative values of $c$ and $m_{f}$. Following this reasoning, in section 5 we consider the nonlinear action in terms of $g_{\mu \nu}$ and the nonlinear massive mode $M_{\mu \nu}$.

\section{The nonlinear massless and massive modes}

Now we consider extending the mass eigenstates of linearized bimetric theory to nonlinear fields. The ADM analysis of the bimetric action shows that even nonlinearly the theory has seven propagating modes [17]. But only their linear fluctuations around $\bar{f}_{\mu \nu}=c^{2} \bar{g}_{\mu \nu}$ backgrounds combine into well defined massless and massive spin-2 states. Here we explore the nonlinear extensions of these mass eigenstates. In a theory with general covariance, spin2 fields are minimally represented by rank- 2 symmetric tensors. Below we find such tensors that reduce to the mass eigenstates (3.6) and (3.7) at the linear level. Since this choice is not unique, one can also invoke simplicity as a criterion. These are the only criteria employed here. We have not considered if the nonlinear modes also propagate two, respectively, five degrees of freedom at the nonlinear level.

\subsection{The nonlinear massless spin-2 field $G$}

The nonlinear massless mode is a symmetric rank-2 tensor $G_{\mu \nu}$ that reproduces the massless fluctuation $\delta G(3.6)$ at the linear level. To determine $G_{\mu \nu}$ we work with the $(1,1)$ tensor,

$$
S_{\nu}^{\mu}=\left(\sqrt{g^{-1} f}\right)^{\mu}{ }_{\nu} .
$$

\footnotetext{
${ }^{6}$ Equally well, one could replace $g_{\mu \nu}$ by $f_{\mu \nu}$ and $c$ by $1 / c$
} 
First note that $\sqrt{g^{-1} f}=g^{-1}\left(\sqrt{f g^{-1}}\right) g$. This follows on writing $\sqrt{g^{-1} f}=\sqrt{\mathbb{1}+\left(g^{-1} f-\mathbb{1}\right)}$ and formally expanding the square-root. We then have the important property, ${ }^{7}$

$$
g S=S^{T} g
$$

Now, let us start with a general symmetric $(0,2)$ tensor $G_{\mu \nu}(g, f)$ and, in it, replace $f$ by $S$ through $f=g S^{2}$. In general, $G_{\mu \nu}$ could contain powers of $S_{\nu}^{\mu},\left(S^{-1}\right)_{\nu}^{\mu},\left(S^{T}\right)_{\nu}^{\mu}$ and $\left(S^{-1 T}\right)_{\nu}^{\mu}$ contracted with $g_{\mu \nu}$ in the right way to produce a $(0,2)$ tensor. Then using $(4.2)$ it is easy to see that general covariance alone restricts $G_{\mu \nu}$ to the form,

$$
G=g \Phi(S)
$$

where $\Phi_{\nu}^{\mu}$ is a matrix function of the matrix $S_{\nu}^{\mu}$ and its inverse, but not its transpose. On the proportional backgrounds $\bar{f}=c^{2} \bar{g}$, where $\bar{S}_{\nu}^{\mu}=c \delta_{\nu}^{\mu}$, this becomes,

$$
\bar{G}=\bar{g} \bar{\Phi} \equiv \bar{g} \Phi(\bar{S}=c \mathbb{1}) .
$$

Clearly, $\bar{\Phi}=\phi(c) \mathbb{1}$ for a scalar $\phi(c) . \bar{\Phi}(\bar{S})$ depends on $c$ in two ways: through an explicit dependence of $\Phi$ on $c$ (e.g., through normalizations), and through $\bar{S}$. If these two types of contributions could be disentangled, $\Phi$ could be uniquely reconstructed from $\bar{\Phi}$.

Let us now consider fluctuations $G_{\mu \nu}=\bar{G}_{\mu \nu}+\delta G_{\mu \nu}^{\prime}$. These can be computed using the canonically normalized variables of the previous section. But to ensure explicitly that the equations depend on $c$ only through $\bar{S}$ and not through normalizations, here we work with,

$$
g_{\mu \nu}=\bar{g}_{\mu \nu}+\delta g_{\mu \nu}^{\prime}, \quad f_{\mu \nu}=\bar{f}_{\mu \nu}+\delta f_{\mu \nu}^{\prime}, \quad \delta S_{\nu}^{\mu}=\frac{1}{2} \bar{g}^{\mu \lambda}\left(\frac{1}{c} \delta f_{\lambda \nu}^{\prime}-c \delta g_{\lambda \nu}^{\prime}\right),
$$

Then the fluctuation of the nonlinear massless field becomes,

$$
\begin{aligned}
\delta G_{\mu \nu}^{\prime} & =\delta g_{\mu \lambda}^{\prime} \bar{\Phi}_{\nu}^{\lambda}+\left.\bar{g}_{\mu \lambda} \frac{\partial \Phi_{\nu}^{\lambda}}{\partial S_{\beta}^{\alpha}}\right|_{\bar{S}} \delta S_{\beta}^{\alpha}+\cdots \\
& =\delta g_{\mu \lambda}^{\prime} \bar{\Phi}_{\nu}^{\lambda}-\left.\frac{c}{2} \bar{g}_{\mu \lambda} \frac{\partial \Phi_{\nu}^{\lambda}}{\partial S_{\beta}^{\alpha}}\right|_{\bar{S}} \bar{g}^{\alpha \sigma} \delta g_{\sigma \beta}^{\prime}+\left.\frac{1}{2 c} \bar{g}_{\mu \lambda} \frac{\partial \Phi_{\nu}^{\lambda}}{\partial S_{\beta}^{\alpha}}\right|_{\bar{S}} \bar{g}^{\alpha \sigma} \delta f_{\sigma \beta}^{\prime}+\cdots
\end{aligned}
$$

On the other hand, in terms of (4.5) the massless fluctuation (3.6) becomes,

$$
\delta G_{\mu \nu}^{\prime}=A\left(\delta g_{\mu \nu}^{\prime}+\alpha^{2} \delta f_{\mu \nu}^{\prime}\right)
$$

with a normalization $A$. Comparing the coefficients of the fluctuations in (4.6) and (4.7) gives two equations for $\Phi$ as a function of $S$, evaluated at $\bar{S}=c \mathbb{1}$,

$$
A^{-1} \bar{\Phi}_{\nu}^{\lambda}=\left(1+\alpha^{2} c^{2}\right) \delta_{\nu}^{\lambda},\left.\quad A^{-1} \frac{\partial \Phi_{\nu}^{\lambda}}{\partial S_{\beta}^{\alpha}}\right|_{c 1}=2 c \alpha^{2} \delta_{\alpha}^{\lambda} \delta_{\nu}^{\beta} .
$$

\footnotetext{
${ }^{7}$ From the above properties of $S^{\mu}{ }_{\nu}$ it follows that, $f=S^{\mathrm{T}} g S$. Hence, $S$ is a local transformation between $f_{\mu \nu}$ and $g_{\mu \nu}$, or a generalized vielbein. Further, in terms of $S$ the proportional backgrounds (2.11) are characterized by the background value $\bar{S}_{\nu}^{\mu}=c \delta^{\mu}{ }_{\nu}$ which is invariant under general coordinate transformations.
} 
The right-hand sides acquire their $c$-dependence only through $\bar{S}=c \mathbb{1}$, and not normalizations. It is then natural to assume that $A^{-1} \Phi(S)$ depends on $c$ only through $\bar{S}=c \mathbb{1}$. This leads to the unique solution,

$$
\Phi=A\left(\mathbb{1}+\alpha^{2} S^{2}\right)
$$

obtained from the first equation on replacing $c$ by $S$. It gives the nonlinear massless mode,

$$
G_{\mu \nu}=A\left(g_{\mu \nu}+\alpha^{2} f_{\mu \nu}\right)
$$

Without loss of generality we can set $A=1$. The fluctuations of this mode can be canonically normalized either with respect to $\bar{g}$, to give (3.6), or with respect to $\bar{G}_{\mu \nu}$.

Other nonlinear extensions of $\delta G_{\mu \nu}$ can be found if $A^{-1} \Phi(S)$ is allowed to have an explicit dependence on $c$, besides that coming from $\bar{S}$. To find these note that,

$$
\left.\frac{\delta\left(S^{n}\right)_{\nu}^{\lambda}}{\delta S_{\beta}^{\alpha}}\right|_{c 1}=n c^{n-1} \delta_{\alpha}^{\lambda} \delta_{\nu}^{\beta}
$$

Then general c-dependent solutions of (4.8) can be written as,

$$
\Phi=A\left(a_{0}+2 \alpha^{2} \sum_{n>0} a_{n} c^{2-n} S^{n}\right), \quad \text { with } \quad a_{0}+2 \alpha^{2} c^{2} \sum_{n>0} a_{n}=1+\alpha^{2} c^{2}, \quad \sum_{n>0} n a_{n}=1 .
$$

Of course, an infinite number of such solutions exists, the one with the lowest power of $S$ being $A^{-1} \Phi=1-\alpha^{2} c^{2}+2 \alpha^{2} c S$. A non-polynomial solution of (4.8) is $A^{-1} \Phi=\left(1+\alpha^{2} c^{4} S^{-2}\right)^{-1}$, giving the massless mode $G^{-1}=g^{-1}+\alpha^{2} c^{4} f^{-1}$.

Of all these, the c-independent solution (4.10) gives the simplest invertible relation between the nonlinear modes and the original bimetric variables $g$ and $f$. Note that to identify this unique c-independent mode, it was important to work with $c \neq 1$ backgrounds. Otherwise, at $c=1$, this criterion is not useful.

\subsection{The nonlinear massive spin-2 field $M$}

From the outset it is evident that the nonlinear massive field is closely related to $S_{\nu}^{\mu}=$ $\left(\sqrt{g^{-1} f}\right)_{\nu}^{\mu}$. This is hinted by the linear equations $(3.2),(3.7)$ and also by the fact that the mass potential $V$ in $(2.2)$ is a polynomial in $S . S^{\mu}{ }_{\nu}$ is a $(1,1)$ tensor but it can be brought to a symmetric $(0,2)$ form in more than one way and the nonlinear extensions of the massive spin-2 fluctuation are related to these $(0,2)$ forms. Here we consider two nonlinear extensions, $M_{\mu \nu}$ and $M_{\mu \nu}^{G}$, before discussing the general case.

In terms of $S_{\mu \nu} \equiv g_{\mu \lambda} S_{\nu}^{\lambda}$, equation (4.2) is the symmetry condition,

$$
S_{\mu \nu}=S_{\nu \mu}
$$

The fluctuation $\delta\left(S_{\mu \nu}\right)=c \delta g_{\mu \nu}+\tilde{c} \delta M_{\mu \nu}$ is a mixture of $\delta g$ and the massive mode $\delta M(3.7)$. Now it is obvious that a nonlinear massive mode can be written as,

$$
M_{\mu \nu}=B\left(g_{\mu \lambda} S_{\nu}^{\lambda}-c g_{\mu \nu}\right),
$$


allowing for a normalization $B$. On proportional backgrounds, $\bar{M}_{\mu \nu}=0$. This is a natural vacuum value for a non-gravitational spin-2 field in the sense that it does not break general covariance in its vacuum. Fluctuations around this background are the massive modes $\delta M$ (3.7) with a Fierz-Pauli mass term. ${ }^{8}$ The condition $g S=S^{T} g$ implies that $S^{\mu}{ }_{\nu}$ and $g_{\mu \nu}$ are not independent fields, whereas $M_{\mu \nu}$ and $g_{\mu \nu}$ can be regarded as independent.

A different nonlinear extension of the massive fluctuation is obtained by using $G_{\mu \nu}(4.10)$ instead of $g_{\mu \nu}$,

$$
M_{\mu \nu}^{G}=\frac{B}{A\left(1+\alpha^{2} c^{2}\right)}\left(G_{\mu \lambda} S_{\nu}^{\lambda}-c G_{\mu \nu}\right)
$$

The normalization is fixed such that $\delta M^{G}=\delta M$. It is easier to invert the relations and express $(g, f)$ in terms of $\left(G, M^{G}\right)$ rather than in terms of $(G, M)$. For more on this see Appendix C.

In general, the massive fluctuation $\delta M$ has many possible nonlinear extensions. More nonlinear extensions can be obtained by following a procedure similar to the massless case. By general covariance alone, any matrix function $M$ of $g$ and $f$ can be written as

$$
M=g \Psi(S) .
$$

The fluctuations of this field,

$$
\delta M_{\mu \nu}=\delta g_{\mu \lambda}^{\prime} \bar{\Psi}_{\nu}^{\lambda}+\left.\bar{g}_{\mu \lambda} \frac{\partial \Psi_{\nu}^{\lambda}}{\partial S_{\beta}^{\alpha}}\right|_{\bar{S}} \delta S_{\beta}^{\alpha}+\cdots
$$

should be equated to the massive fluctuation (3.7) with arbitrary normalization $B$,

$$
\delta M_{\mu \nu}=B \bar{g}_{\mu \lambda} \delta S_{\nu}^{\lambda}
$$

This gives,

$$
B^{-1} \bar{\Psi}=\Psi(c \mathbb{1})=0,\left.\quad B^{-1} \frac{\partial \Psi_{\nu}^{\lambda}}{\partial S_{\beta}^{\alpha}}\right|_{\bar{S}}=\delta_{\alpha}^{\lambda} \delta_{\nu}^{\beta} .
$$

Again the $c$-dependence of the right-hand sides comes only from $\bar{S}$ and not from normalizations. However, now we cannot assume that $B^{-1} \Psi$ depends on $S$ and not explicitly on $c$ since then $\bar{\Psi}(c)=0$ would imply $\Psi(S)=0$, identically. At a least a minimal $c$-dependence is needed to get a nonlinear massive mode with a vanishing background value. The solution with the simplest and most natural $c$ dependence is the one corresponding to (4.14),

$$
\Psi_{\nu}^{\lambda}=B\left(S_{\nu}^{\lambda}-c \delta_{\nu}^{\lambda}\right)
$$

More general solutions of equations (4.19), involving higher powers of $S$, are given by,

$$
\Psi=B \sum_{n \geq 0} b_{n} c^{1-n} S^{n}, \quad \text { with } \quad \sum_{n \geq 0} b_{n}=0, \quad \sum_{n \geq 1} n b_{n}=1 .
$$

For example, one can check that the massive mode $M^{G}(4.15)$ is a solution to the above equations by reading off the $b_{n}$ from $B^{-1} M^{G}=\left(1+c^{2} \alpha^{2}\right)^{-1} g\left(-c+S-c \alpha^{2} S^{2}+\alpha^{2} S^{3}\right)$.

\footnotetext{
${ }^{8}$ A note on notation: for $c=1$ and $f_{\mu \nu}=\eta_{\mu \nu}, g^{-1} M$ coincides with the $K=\sqrt{g^{-1} \eta}-1$ in terms of which the dRGT model is written. $K_{\nu}^{\mu}$ was engineered to produce the massive mode around flat space for $c=1$, while $M$ represents the massive mode around any background for which a Fierz-Pauli mass can be written.
} 


\subsection{Absence of ghost-free matter coupling of the massless mode $G$}

In the previous subsection we obtained a nonlinear generalization $G_{\mu \nu}$ (4.10) of the massless fluctuation of bimetric theory. It is natural to ask if $G_{\mu \nu}$ could be consistently coupled to matter in the standard way, and be interpreted as the gravitational metric. As in GR, such matter couplings must be consistent with the weak equivalence principle. Here we show that minimal couplings of $G_{\mu \nu}$ are not ghost-free. The alternative, then, is to regard $g_{\mu \nu}$ as the gravitational metric and rely on the weak gravity limit discussed earlier.

Consider standard minimal couplings of $G_{\mu \nu}$ to matter, for example, to a scalar field $\phi$,

$$
\mathcal{L}_{(m, G)}=-\sqrt{-G} G^{\mu \nu} \partial_{\mu} \phi \partial_{\nu} \phi
$$

To see if such ghost-free couplings exist in the bimetric theory, one can perform a Hamiltonian (ADM) analysis [36]. We introduce the following notation for the $3+1$ decomposition of $G$,

$$
G_{\mu \nu}=\left(\begin{array}{cc}
-K^{2}+K^{l} K_{l} & K_{j} \\
K_{i} & { }^{3} G_{i j}
\end{array}\right)
$$

where $K_{i}={ }^{3} G_{i j} K^{j}$. Standard matter couplings of $G_{\mu \nu}$ such as (4.22), when written in the Hamiltonian form using canonically conjugate variables, are linear in $K$ and $K_{i}$,

$$
\mathcal{L}_{(m, G)}=\tilde{\mathcal{L}}+K \tilde{\mathcal{C}}+K_{i} \tilde{\mathcal{C}}^{i}
$$

If the dynamics of $G_{\mu \nu}$ were described by the Einstein-Hilbert action, $\sqrt{-G} R_{G} \sim \Pi^{i j} \partial_{t}{ }^{3} G_{i j}+$ $K \mathcal{C}+K_{i} \mathcal{C}^{i}$, then $K$ and $K_{i}$ would be Lagrange multipliers in the full theory. Their equations of motion would result in four constraints that, along with gauge symmetries, would eliminate the ghost and leave two propagating modes for $G_{\mu \nu}$. However, in bimetric theory, the nonlinear action expressed in terms of $G_{\mu \nu}$ and $M$ (or $M^{G}$ ) is complicated and it is not convenient to carry out the ghost analysis in terms of the ADM variables of $G_{\mu \nu}$. Instead, since the bimetric analysis is already known in terms of the ADM variables for $g$ and $f$ [17], the strategy here is to analyze the matter coupling (4.24) in terms of these variables.

To this end, we introduce the $3+1$ decompositions of $g$ and $f$,

$$
g_{\mu \nu}=\left(\begin{array}{cc}
-N^{2}+N^{l} N_{l} & N_{j} \\
N_{i} & { }^{3} g_{i j}
\end{array}\right), \quad f_{\mu \nu}=\left(\begin{array}{cc}
-L^{2}+L^{l} L_{l} & L_{j} \\
L_{i} & { }^{3} f_{i j}
\end{array}\right) .
$$

The indices on $N_{i}$ and $L_{i}$ are raised using the inverses of ${ }^{3} g_{i j}$ and ${ }^{3} f_{i j}$, respectively. It is known that in terms of new variables $n^{i}$ that parameterize $N^{i}-L^{i}$ through (for details and the form of the matrix $D$, see $[17,21])$,

$$
N^{i}=L^{i}+L n^{i}+N D^{i} n^{k}
$$

the bimetric theory (2.1) (with no matter couplings) takes the form [17],

$$
\mathcal{L}=m_{\mathrm{eff}}^{2}\left[\pi^{i j} \partial_{t} g_{i j}+p^{i j} \partial_{t} f_{i j}+N \mathcal{C}_{g}+L \mathcal{C}_{f}+L_{i} R_{g f}^{i}\right] .
$$


For convenience we use the scaled fields of appendix B, effectively setting $m_{g}=m_{f} . \pi_{i j}$ and $p_{i j}$ are the momenta conjugate to $g_{i j}$ and $f_{i j}$. The functions $\mathcal{C}_{g}, \mathcal{C}_{f}$ and $R_{g f}^{i}$ are independent of $N, L$ and $L_{i}$, but depend on $n^{i}$ and the remaining variables. The action has the additional property that the $n^{i}$ equations of motion are independent of $N, L$ and $L_{i}$, and determine $n^{i}$ in terms of the remaining variables. Thus, $N, L$ and $L_{i}$ are five Lagrange multipliers whose equations, in particular, $\mathcal{C}_{g}=0$ and $\mathcal{C}_{f}=0$, provide the constraints that render the theory ghost-free (along with the associated secondary constraints and gauge conditions) [17, 18].

To emphasize, this argument for the absence of ghosts crucially depends on the possibility of parameterizing the $N^{i}$ in terms of the $n^{i}$ through (4.26). Only then $N, L$ and $L_{i}$ appear linearly in the action (4.27) and enforce the required constraints. Introducing standard matter couplings for $g_{\mu \nu}$ and $f_{\mu \nu}$ individually, does not change this story. ${ }^{9}$ If, instead, one couples a combination of $g$ and $f$ to matter, one has to insure that it does not reintroduce ghosts by destroying the constraints.

Now, we consider the matter coupling of the nonlinear massless mode $G=g+f$ by adding (4.24) to (4.27). The relevant terms in the action are,

$$
N \mathcal{C}_{g}+L \mathcal{C}_{f}+L_{i} R_{g f}^{i}++K \tilde{\mathcal{C}}+K_{i} \tilde{\mathcal{C}}^{i}
$$

From the $3+1$ decompositions of $G_{\mu \nu}, g_{\mu \nu}$ and $f_{\mu \nu}$ it is easy to see that,

$$
\begin{aligned}
K_{i} & =N_{i}+L_{i}, \quad{ }^{3} G_{i j}={ }^{3} g_{i j}+{ }^{3} f_{i j}, \\
K^{2} & =N^{2}+L^{2}+{ }^{3} G^{i j} K_{i} K_{j}+-{ }^{3} g_{i j} N^{i} N^{j}-{ }^{3} f_{i j} L^{i} L^{j} .
\end{aligned}
$$

Already at first glance $K$ is highly nonlinear in $N$ and $L$ which are no longer Lagrange multipliers. But this may not yet imply a ghost. Note that after the $N^{i}$ have been expressed in terms of the $n^{i}$ (4.26), we may still carry out a similar reparameterization of the $L^{i}$ in terms of some $l^{i}$. If this could somehow render (4.28) linear in $N$ and $L$, then the theory may still have the constraints to avoid ghosts (although it may propagate more than seven modes if the $L^{i}$ constraints are lost). But it turns out that $K^{2}$ given above is independent of $L_{i}$, so reparameterizing it does not help.

To see this, simplify the expression for $K^{2}$ using (4.29) and writing $N^{i}=\left(N^{i}-L^{i}\right)+L^{i}$,

$$
K^{2}=N^{2}+L^{2}+\left(N^{i}-L^{i}\right)\left(N^{j}-L^{j}\right)\left({ }^{3} g_{i j}-{ }^{3} g_{i k}{ }^{3} G^{k l}{ }^{3} g_{l j}\right) .
$$

Since $N^{i}-L^{i}=L n^{i}+N D_{k}^{i} n^{k}$, in terms of $n^{i}$, this is independent of $L^{i}$ and has the form,

$$
K^{2}=c_{1} N^{2}+c_{2} L^{2}+2 c_{3} L N .
$$

For the given $c_{1}, c_{2}$ and $c_{3}$, this expression is not a prefect square implying that there is no way to render $K$ linear in $N$ and $L$. Hence the associated constraints are lost. Therefore, coupling $G_{\mu \nu}$ to matter will reintroduce ghosts. Of course, to linear order, $\delta K$ is linear in $\delta N$ and $\delta L$ and, to this order, ghost-free matter couplings exist, as in (3.8).

\footnotetext{
${ }^{9}$ Coupling $g_{\mu \nu}$ and $f_{\mu \nu}$ individually to matter in the standard way, as in (1.2), results in terms of the form (4.24), now written for the metrics $g$ and $f$. Adding these to the bimetric action (4.27) simply modifies $\mathcal{C}_{g}, \mathcal{C}_{f}$ and $R_{g f}^{i}$, but keeps the Lagrange multipliers. Hence the no-ghost argument goes through unmodified.
} 


\subsection{Spin-2 mixing and oscillations}

In the absence of ghost-free coupling of the massless mode $G_{\mu \nu}$ to matter, one is led to consider the standard individual couplings of $g_{\mu \nu}$ and $f_{\mu \nu}$ to matter, which are known to be ghost-free. In the weak gravity limit, $m_{g}>>m_{f}$, we regard $g_{\mu \nu}$ as the gravitational metric. The fluctuations $\delta g$ and $\delta f$, sourced respectively by $\delta T^{g}$ and $\delta T^{f}$, are linear combinations of the mass eigenstates $\delta G$ and $\delta M$ given in (3.6) and (3.7). So the spin-2 states are produced in the interaction basis $(\delta g, \delta f)$ while they propagate as mass eigenstates $(\delta M, \delta G)$. As is well known, this will lead to oscillations between $(\delta g, \delta f)$ and a graviton $\delta g$ may oscillate to the other spin-2 field $\delta f$. This is very similar to neutrino oscillations or the $K_{0}-\bar{K}_{0}$ oscillations. The detectability of this effect reduces for higher FP mass of the massive mode. So in cases where the massive spin-2 state can be interpreted as a meson or a heavy elementary particle, the effect is negligible. But it will have consequences for very light spin-2 states. Nevertheless it remains an interesting consequence of the inconsistency of coupling the massless field to matter that in the presence of a neutral massive spin-2 field, the gravitational force is mediated by a particle that is a superposition of mass eigenstates.

\section{Action for the nonlinear massive spin-2 field}

In this section we consider the bimetric action (2.1) as a theory of a massive spin-2 field $M_{\mu \nu}$ in the presence of a gravitational metric $g_{\mu \nu}$. We also obtain the ghost-free couplings of $M_{\mu \nu}$ to fermionic matter fields.

\subsection{The action in terms of $g$ and $M$}

To regard the bimetric action (2.1) as a theory of a massive spin-2 field in the presence of gravity, we express it in terms of the nonlinear massive field $M_{\mu \nu}$ and the metric $g_{\mu \nu} \cdot{ }^{10} g_{\mu \nu}$ couples to matter in the standard way and is the gravitational metric. $M_{\mu \nu}$ is a massive spin-2 field and couples non-minimally to gravity. The two spin-2 fields mix and their mass and interaction eigenstates do not coincide, just as for spin- $\frac{1}{2}$ fields in the standard model. These mixings also result in deviations from GR. In section 3, in the linearized theory, the mixings became small in the weak gravity limit $m_{g}>>c m_{f}$. The hope is that in this limit the mixings remain small even nonlinearly and the predictions of this theory do not greatly differ from GR.

To express the bimetric action (2.1) in terms of the fields $g$ and the massive spin-2 field $M$, let us start with the potential $V\left(S, \beta_{n}\right)$, where $S=\sqrt{g^{-1} f}(2.2)$, and use,

$$
S_{\nu}^{\rho}=g^{\rho \sigma} M_{\sigma \nu}+c \delta_{\nu}^{\rho} .
$$

\footnotetext{
${ }^{10}$ Other possibilities would be to write the bimetric action in terms of the nonlinear massless field $G_{\mu \nu}$ (4.10) and the massive field $M$ or $M^{G}$. The $G-M^{G}$ action is given in the appendix. In the $G-M$ case the expressions for $g$ and $f$ become too involved. Since $G_{\mu \nu}$ cannot be coupled to matter in a ghost-free way, here we concentrate on the $g-M$ case.
} 
Then, the potential written in terms of elementary symmetric polynomials, becomes [19],

$$
V\left(S, \beta_{n}\right)=\sum_{n=0}^{4} \beta_{n} e_{n}(S)=\sum_{n=0}^{4} \beta_{n} c^{n} e_{n}\left(\mathbb{1}+g^{-1} M / c\right)=\sum_{n=0}^{4} \alpha_{n}^{c} e_{n}\left(g^{-1} M\right)=V\left(g^{-1} M, \alpha_{n}^{c}\right)
$$

The last step follows from the linear relations between the $e_{n}(\mathbb{X})$ and $e_{n}(\mathbb{1}+\mathbb{X}) .{ }^{11}$ The parameters $\alpha_{n}^{c}$ are given in terms of $\beta_{n}$ as,

$$
\begin{array}{lrl}
\alpha_{4}^{c}=\beta_{4}, & \alpha_{3}^{c}=\beta_{3}+c \beta_{4}, & \alpha_{2}^{c}=\beta_{2}+2 c \beta_{3}+c^{2} \beta_{4}, \\
\alpha_{1}^{c}=\beta_{1}+3 c \beta_{2}+3 c^{2} \beta_{3}+c^{3} \beta_{4}, & \alpha_{0}^{c}=\beta_{0}+4 c \beta_{1}+6 c^{2} \beta_{2}+4 c^{3} \beta_{3}+c^{4} \beta_{4} .
\end{array}
$$

An advantage of writing the theory in terms of $M$, of course, is that the potential no longer involves a square-root matrix. Also, this form is analogous to the familiar form of mass terms in field theory, for example, the mass term for massive vector fields, $\sqrt{-g} g^{\mu \nu} A_{\mu} A_{\nu}$.

Now let us turn to the kinetic term for the massive field $M$ which is obtained from $\sqrt{-f} R(f)$ on expressing $f$ in terms of $M$ and $g$. The criterion is that in the final action for $M_{\mu \nu}$, all covariant derivatives must be with respect to the metric $g_{\mu \nu}$. To achieve this in a systematic way, it is convenient to use,

$$
f_{\mu \nu}=g_{\mu \rho}\left(S^{2}\right)_{\nu}^{\rho}
$$

where $S$ is related to $M$ in a simple way (5.1). Now, the curvatures of $f$ can be expressed in terms of curvatures of $g$ using the results in appendix A. In particular, (A.3) gives,

$$
R_{\mu \nu}(f)=R_{\mu \nu}(g)+2 \nabla_{[\mu} C_{\alpha] \nu}{ }^{\alpha}-2 C_{\nu[\mu}{ }^{\beta} C_{\alpha] \beta}{ }^{\alpha},
$$

where $\nabla$ is the covariant derivative compatible with $g_{\mu \nu}$ and,

$$
C_{\mu \nu}^{\alpha}=\frac{1}{2} \phi^{\alpha \beta}\left(\nabla_{\mu} f_{\beta \nu}+\nabla_{\nu} f_{\beta \mu}-\nabla_{\beta} f_{\mu \nu}\right) .
$$

Here, $f$ is given by (5.3) and, for ease of notation we have introduced,

$$
\phi^{\mu \nu} \equiv\left(f^{-1}\right)^{\mu \nu}=\left(S^{-2}\right)_{\rho}^{\mu} g^{\rho \nu} .
$$

Using the curvature relation above, along with $\sqrt{-f}=\sqrt{-g} \operatorname{det}(S)$ and $R(f)=\phi^{\mu \nu} R_{\mu \nu}(f)$, it is a straightforward though tedious exercise to show that (modulo total derivatives),

$$
\sqrt{-f} R(f)=\sqrt{-g} \operatorname{det}(S)\left[\phi^{\mu \nu} R_{\mu \nu}(g)+\Pi_{\rho \omega}^{\sigma \pi \alpha \beta} \nabla_{\alpha} S_{\sigma}^{\rho} \nabla_{\beta} S_{\pi}^{\omega}\right] .
$$

The "polarization" tensor $\Pi$ is a function of $g$ and $M$ (through $S$ ) given by,

$$
\Pi_{\rho \omega}^{\sigma \pi \alpha \beta}=\left(2 \phi^{\alpha \mu} \phi^{\kappa \delta} \phi^{\beta \nu}-2 \phi^{\alpha \mu} \phi^{\beta \kappa} \phi^{\delta \nu}-\phi^{\alpha \beta} \phi^{\kappa \delta} \phi^{\mu \nu}+\phi^{\alpha \beta} \phi^{\kappa \mu} \phi^{\delta \nu}\right) \delta_{(\kappa}^{\sigma} \delta_{\delta)}^{\gamma} \delta_{(\mu}^{\pi} \delta_{\nu)}^{\lambda} S_{\gamma \rho} S_{\lambda \omega} .
$$

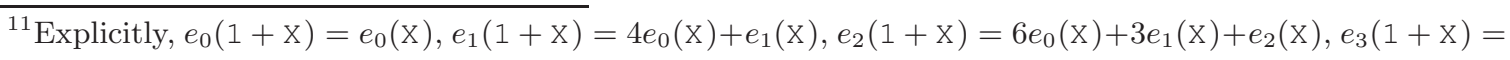
$4 e_{0}(\mathrm{X})+3 e_{1}(\mathrm{X})+2 e_{2}(\mathrm{X})+e_{3}(\mathrm{X})$, and $e_{4}(1+\mathrm{X})=e_{0}(\mathrm{X})+e_{1}(\mathrm{X})+e_{2}(\mathrm{X})+e_{3}(\mathrm{X})+e_{4}(\mathrm{X})$.
} 
The discarded total derivative terms in (5.7) arise since the left-hand side has $f \partial^{2} f$ terms, leading to $M \partial^{2} M$ terms on the right-hand side. These have been converted to $\partial M \partial M$ terms which amounts to adding the Gibbons-Hawking-York boundary term to the action for $f$.

Finally, putting all this together, the action for a massive spin-2 field $M$ interacting with a gravitational metric $g$ is given by,

$$
\begin{aligned}
S_{g M}=\int \mathrm{d}^{4} x \sqrt{-g}[ & m_{g}^{2} R(g)+m_{f}^{2} \operatorname{det}(S) \phi^{\mu \nu} R_{\mu \nu}(g) \\
& \left.+m_{f}^{2} \operatorname{det}(S) \Pi_{\rho \omega}^{\sigma \pi \alpha \beta} \nabla_{\alpha} M_{\sigma}^{\rho} \nabla_{\beta} M_{\pi}^{\omega}-2 m^{4} V\left(g^{-1} M, \alpha_{n}^{c}\right)\right] .
\end{aligned}
$$

Here $S_{\nu}^{\mu}$ is a function of $M_{\mu \nu}$. The coupling of $M$ to matter fields will be discussed below.

The perturbative content of the $(g, M)$ action $(2.1)$ can be discerned easily. The second order action for the fluctuations, $g=\bar{g}+\delta g$ and $M=0+\delta M$, will contain $(\delta g)^{2}, \delta g \delta M$, and $(\delta M)^{2}$ terms. It can be diagonalized in terms of the massless mode $\delta G$ and massive mode $\delta M$, leading to the linearized equations (3.8) and (3.9).

\subsection{Some features of the $g-M$ action}

Equivalence of the two formulations: It is straightforward that the $g$ and $M$ equations of motion obtained from (5.9) imply the bimetric $g$ and $f$ equations of motion and vice versa,

$$
\left.\frac{\delta S_{g f}}{\delta f}\right|_{g}=\left.\frac{\delta S_{g M}}{\delta M}\right|_{g} \frac{\delta M}{\delta f}=0,\left.\quad \frac{\delta S_{g f}}{\delta g}\right|_{f}=\left.\frac{\delta S_{g M}}{\delta g}\right|_{M}+\left.\frac{\delta S_{g M}}{\delta M}\right|_{g} \frac{\delta M}{\delta g}=0 .
$$

Hence the $g-M$ formulation is classically equivalent to the $g-f$ formulation and, in particular, is also ghost-free. The $g-M$ form has the advantage that it does not involve square-root matrices. The price one pays on the other hand, is the tedious kinetic structure for $M$ and its kinetic mixing with gravity. Although deriving the equations of motion from (5.9) is not convenient, it is much easier to obtain these equations by starting with the $g-f$ equations (2.6) and (2.7), eliminating $R(f)$, and then converting $R_{\mu \nu}(f)$ to $R_{\mu \nu}(g)$ using (5.4).

In the $g-M$ action, one may perform perturbative calculations even around non-vanishing $M$ backgrounds. In the $g-f$ formulation, performing higher order perturbative calculations

around non-proportional backgrounds is not straightforward as in that case expanding $\sqrt{g^{-1} f}$ is not simple. Of course, the two formulations are not expected to be equivalent in quantum theory unless one takes into account the Jacobian factor that arises from the change of variables.

Parameterizing deviations from general relativity: Most of the classical solutions of the bimetric action $S_{g f}$ do not coincide with classical solutions in general relativity [26-32], except for the class of proportional backgrounds $\bar{f}=c^{2} \bar{g}$ considered here, with $c$ determined by the parameters of the theory. Generic matter couplings of the $g$ and $f$ metrics will drive the solutions away from proportional backgrounds. We are interested in parameter regions where these deviations are small.

In the $g-M$ formulation, proportional backgrounds correspond to $\bar{M}_{\mu \nu}=0$. Hence, in the field theory language, a vanishing vacuum expectation value for the massive spin-2 field 
implies that the classical solutions for the metric coincides with general relativity. Hence, deviations of the bimetric theory from general relativity are parameterized by the deviations of $M_{\mu \nu}$ from zero. These are driven by general couplings of $g_{\mu \nu}$ and $M_{\mu \nu}$ to matter fields that violate the condition $\bar{T}^{f}=\alpha^{2} \bar{T}^{g}(2.15)$.

Energy-momentum tensor of spin-2 fields: Consider the gravitational energy momentum tensor $\delta S_{g f} / \delta g_{\mu \nu}$. In the $g-f$ formulation, $f_{\mu \nu}$ contributes to this only through the potential $V$, but its kinetic term $\sqrt{-f} R(f)$ does not gravitate (of course, it still affects the dynamics of $g_{\mu \nu}$ since their equations of motion are coupled). This is while the Noether energy momentum tensor computed around flat $f_{\mu \nu}$, will receive contributions from $\sqrt{-f} R(f)$.

In the $g-M$ formulation, the gravitational energy momentum tensor $\delta S_{g M} /\left.\delta g_{\mu \nu}\right|_{M}$ contains contributions from the kinetic term of $M$, as well from $V$ and these appear consistent with the contribution to the Noether energy momentum tensor around flat $g$. Of course the complete set of equations is the same in both formalisms as these contributions drop out on imposing the $M$ equation of motion. The same statements apply to the matter couplings of $f$, that is $S(f, \psi)$. In the $g-M$ formulation, $M$ is mostly minimally coupled to $g$ (in the sense that the curved space form can be constructed from the flat space expression) except for the non-minimal $\phi^{\mu \nu} R_{\mu \nu}$ term.

Comparison to earlier work: The $g-M$ action (5.9) is useful in comparing to earlier attempts of writing a theory of massive spin- 2 on a gravitational background. For example, the approach in $[7,8]$ was to start with the quadratic FP theory in flat spacetime and covariantize it with a metric $g_{\mu \nu}$, also adding non-minimal curvature couplings. This procedure will in general not reproduce the action (5.9) as it will miss the factor $\operatorname{det}(S)$ as well as the complicated polarization structure (5.8), since it only considers terms quadratic in the massive field.

\subsection{Coupling massive spin-2 fields to matter}

At present, the only known ghost-free matter couplings in bimetric theory are the standard couplings of $g_{\mu \nu}$ and $f_{\mu \nu}$ to matter sources, as in (1.2). In the weak gravity limit, we interpreted the $g_{\mu \nu}$ couplings as the gravitational interactions of matter fields just as in GR. Then the $f_{\mu \nu}$ couplings give rise to very specific interactions of the massive spin-2 field with matter, dictated by the absence of ghost. To write $f$ in terms of $M$, again it is convenient to proceed through the related matrix $S=g^{-1} M-c \mathbb{1}(5.1)$ and use,

$$
f_{\mu \nu}=S_{\mu}^{\alpha} g_{\alpha \beta} S^{\beta}{ }_{\nu}=g_{\mu \alpha} S_{\beta}^{\alpha} S^{\beta}{ }_{\nu}
$$

For $f_{\mu \nu}$ couplings to bosonic matter, the manipulations are straightforward. For example, for a Proca field, $\mathcal{L}(f, A)=-\frac{1}{4} \sqrt{f}\left[f^{\mu \nu} f^{\kappa \lambda} F_{\mu \kappa} F_{\nu \lambda}+2 m_{A}^{2} f^{\mu \nu} A_{\mu} A_{\nu}\right]$, where $F_{\mu \nu}=\partial_{\mu} A_{\nu}-\partial_{\nu} A_{\mu}$, One obtains the coupling of $A_{\mu}$ to the massive spin-2 field by expressing $f$ in terms of $M$ and $g$ through $S$. Then, on raising some indices using $g^{\mu \nu}$ one gets,

$$
\mathcal{L}(g, M, A)=-\frac{1}{4} \sqrt{g} \operatorname{det} S\left[\left(S^{-2}\right)^{\mu}{ }_{\rho}\left(S^{-2}\right)_{\sigma}^{\kappa} F_{\mu \kappa} F^{\rho \sigma}+2 m_{A}^{2}\left(S^{-2}\right)^{\mu}{ }_{\rho} A_{\mu} A^{\rho}\right] .
$$


The details of this Lagrangian can be investigated further by, e.g., considering flat space $g=\eta$, and/or expanding $S_{\nu}^{\mu}=c \delta_{\nu}^{\mu}+a \bar{g}^{\mu \lambda} \delta M_{\lambda \nu}$.

For fermionic matter a little more work is needed since fermions couple to spin-2 fields through vielbeins. For the $f_{\mu \nu}$ and $g_{\mu \nu}$ vielbeins (below we use the conventions of [53]),

$$
f_{\mu \nu}=\tilde{e}_{\mu}^{a} \eta_{a b} \tilde{e}_{\nu}^{b}, \quad g_{\mu \nu}=e_{\mu}^{a} \eta_{a b} e^{b}{ }_{\nu}
$$

equation (5.11) implies the relation,

$$
\tilde{e}_{\mu}^{a}=\Lambda_{b}^{a} e^{b}{ }_{\nu} S_{\mu}^{\nu}
$$

Here $\Lambda$ is an arbitrary Lorentz transformation, $\Lambda^{\mathrm{T}} \eta \Lambda=\eta$. In a Lorentz invariant theory $\Lambda$ drops out of all expressions, so we set $\Lambda=\mathbb{1}$ without loss of generality. The vielbeins enter the couplings through the curved space $\gamma$-matrices and through the spin-connections. In terms of the Lorentz frame $\gamma$-matrices $\bar{\gamma}^{a}$, one constructs a pair of curved space $\gamma$-matrices $\tilde{\gamma}^{\mu}=\tilde{e}_{a}^{\mu} \bar{\gamma}^{a}$ and $\gamma^{\mu}=e_{a}^{\mu} \bar{\gamma}^{a}$. They satisfy,

$$
\left\{\bar{\gamma}^{a}, \bar{\gamma}^{b}\right\}=2 \eta^{a b}, \quad\left\{\tilde{\gamma}^{\mu}, \tilde{\gamma}^{\nu}\right\}=2 f^{\mu \nu}, \quad\left\{\gamma^{\mu}, \gamma^{\nu}\right\}=2 g^{\mu \nu}
$$

The inverse of the relation (5.11), with $\Lambda=1$, then implies, ${ }^{12}$

$$
\tilde{\gamma}^{\mu}=\left(S^{-1}\right)_{\nu}^{\mu} \gamma^{\nu}
$$

Fermions also couple to vielbeins through spin connections in Lorentz covariant derivatives acting on them,

$$
\tilde{D}_{\mu}=\partial_{\mu}-\frac{1}{8} \tilde{w}_{\mu}^{a b}\left[\bar{\gamma}_{a}, \bar{\gamma}_{b}\right]
$$

The spin-connection is given in terms of vielbeins and the Christoffel connection through,

$$
\tilde{w}_{\mu}^{a b}=\tilde{e}_{\nu}^{b} \partial_{\mu}\left[\eta^{a c} \tilde{e}_{c}^{\nu}\right]+\tilde{e}^{b}{ }_{\sigma} \eta^{a c} \tilde{e}_{c}{ }^{\nu} \tilde{\Gamma}_{\mu \nu}{ }^{\sigma}
$$

Using (5.14), with $\Lambda=1$, we can rewrite this as,

$$
\tilde{w}_{\mu}^{a b}=\left(e^{b}{ }_{\rho} S^{\rho}{ }_{\nu} \partial_{\mu}\left[\left(e^{a \alpha}\left(S^{-1}\right){ }_{\alpha}^{\nu}\right]+e^{b}{ }_{\lambda} S_{\sigma}^{\lambda} e^{a \alpha}\left(S^{-1}\right)_{\alpha}^{\nu} \tilde{\Gamma}_{\mu \nu}{ }^{\sigma}\right) .\right.
$$

From appendix A, $\tilde{\Gamma}$ is related to the Christoffel connection $\Gamma$ of $g_{\mu \nu}$ by,

$$
\tilde{\Gamma}_{\mu \nu}^{\sigma}=\Gamma_{\mu \nu}^{\sigma}+C_{\mu \nu}{ }^{\sigma}, \quad C_{\mu \nu}^{\sigma}=\frac{1}{2} f^{\sigma \rho}\left(\nabla_{\mu} f_{\rho \nu}+\nabla_{\nu} f_{\mu \rho}-\nabla_{\rho} f_{\mu \nu}\right)
$$

where the covariant derivatives are with respect to $g_{\mu \nu}$, and where $f_{\mu \nu}$ is regarded as a function of $g$ and $M$ through (5.11). Using these relations, it is straightforward to re-express any coupling of $f_{\mu \nu}$ to fermions in terms the massive spin-2 field $M_{\mu \nu}$ and the gravitational metric $g_{\mu \nu}$. The resulting expressions are highly nonlinear in the fields.

\footnotetext{
${ }^{12} \mathrm{~A}$ general $\Lambda$ is absorbed by a Lorentz transformation of the spinors, $\psi^{\prime}=A \psi$ where, $\Lambda_{b}^{a} \bar{\gamma}^{b}=A^{\dagger} \bar{\gamma}^{a} A$.
} 
As an example, consider the couplings to lowest order in the fluctuation $\delta M$ of the massive spin-2 field around the $\bar{M}=0$ background. Then, $S^{\mu}{ }_{\nu}=c \delta_{\nu}^{\mu}+a \bar{g}^{\mu \lambda} \delta M_{\lambda \nu}$, and to first order,

$$
C_{\mu \nu}{ }^{\sigma}=\frac{a}{c} g^{\sigma \rho}\left[2 \nabla_{(\mu} \delta M_{\nu) \rho}-\nabla_{\rho} \delta M_{\mu \nu}\right] .
$$

Similarly, to this order,

$$
\tilde{\gamma}^{\mu}=\frac{1}{c} \gamma^{\mu}-\frac{a}{c^{2}} \bar{g}^{\mu \lambda} \delta M_{\lambda \nu} \gamma^{\nu}, \quad \tilde{D}_{\mu}=D_{\mu}-\frac{a}{4 c}\left[\gamma^{\rho}, \gamma^{\sigma}\right] \nabla_{[\rho} \delta M_{\sigma] \mu} .
$$

Let us apply these to the coupling of $f_{\mu \nu}$ to a spin- $\frac{1}{2}$ field $\psi$,

$$
\mathcal{L}_{1 / 2}=i \sqrt{-f} \bar{\psi}\left(\tilde{\gamma}^{\mu} \tilde{\mathcal{D}}_{\mu}+i m_{\psi}\right) \psi+\text { h.c. },
$$

in which $\bar{\psi}=\psi^{\dagger} \bar{\gamma}^{0}$. $\tilde{\mathcal{D}}_{\mu}=\tilde{D}_{\mu}+i q A_{\mu}$ is the Lorentz and gauge covariant derivative with Abelian gauge field $A_{\mu}$. We write this to linear order in $\delta M$ and in the flat space limit $g_{\mu \nu}=\eta_{\mu \nu}$. Using, $\sqrt{-f}=c^{4}+c^{3} a \delta M_{\rho}^{\rho}+\cdots$, one has,

$$
\begin{aligned}
\mathcal{L}_{1 / 2}=c^{3}(1 & \left.+\frac{1}{c m_{\mathrm{eff}}} \delta M_{\rho}^{\rho}\right) \mathcal{L}_{\text {free }} \\
& -i \frac{c^{3}}{c m_{\mathrm{eff}}} \bar{\psi}\left(\delta M_{\nu}^{\mu} \bar{\gamma}^{\nu} \partial_{\mu}+i q \delta M^{\mu}{ }_{\nu} \bar{\gamma}^{\nu} A_{\mu}+\frac{1}{4} \bar{\gamma}^{\mu}\left[\bar{\gamma}^{\rho}, \bar{\gamma}^{\nu}\right] \partial_{[\rho} \delta M_{\nu] \mu}\right) \psi+\text { h.c. },
\end{aligned}
$$

where $\mathcal{L}_{\text {free }}=i \bar{\psi}\left(\gamma^{\mu} \partial_{\mu}+i q \bar{\gamma}^{\mu} A_{\mu}+i c m_{\psi}\right) \psi$. After a partial integration and using (5.15), the derivative couplings become,

$$
\begin{aligned}
-\frac{i c^{3}}{c m_{\mathrm{eff}}} \delta M_{\mu \nu}\left[\frac{3}{8} \bar{\psi}\left(\gamma^{\mu} \partial^{\nu}+\gamma^{\nu} \partial^{\mu}\right) \psi\right. & -\frac{1}{8}\left(\partial^{\mu} \bar{\psi} \gamma^{\nu}+\partial^{\nu} \bar{\psi} \gamma^{\mu}\right) \psi \\
& \left.+\frac{1}{4} \eta^{\mu \nu} \partial_{\rho} \bar{\psi} \gamma^{\rho} \psi-\frac{3}{4} \eta^{\mu \nu} \bar{\psi} \gamma^{\rho} \partial_{\rho} \psi\right]+ \text { h.c. }
\end{aligned}
$$

Finally, considering the hermitian conjugate (with the usual hermiticity condition $\left(\gamma^{\mu}\right)^{\dagger}=$ $\left.\gamma^{0} \gamma^{\mu} \gamma^{0}\right)$, this can be written,

$$
-\frac{i c^{3}}{c m_{\mathrm{eff}}} \delta M_{\mu \nu}\left[\frac{1}{2} \bar{\psi}\left(\gamma^{\mu} \partial^{\nu}+\gamma^{\nu} \partial^{\mu}\right) \psi-\eta^{\mu \nu} \bar{\psi} \gamma^{\rho} \partial_{\rho} \psi\right]+\text { h.c. . }
$$

Couplings of this form were recently considered in a phenomenological context in [54], to address the top-quark forward-backward asymmetry. Here, in contrast to [54], the couplings are not a priori expected to be flavor violating since they come only from the Lorentz covariant derivative and are essentially of a purely gravitational nature. In particular, the first term of (5.26) is simply the stress-energy tensor while the second corresponds to a non-derivative trace coupling on-shell.

\section{Generalization to more than one massive field}

Recently, in [24] the bimetric action was generalized to a ghost-free theory of $\mathcal{N}$ interacting spin-2 fields. In this theory, the kinetic term is given in terms of $\mathcal{N}$ metrics $g_{\mu \nu}(I)$ and the interactions between these are constructed in terms of the corresponding vielbeins $e_{\mu}^{a}(I)$,

$$
\sum_{I=1}^{\mathcal{N}} \int \mathrm{d}^{4} x \sqrt{-g(I)} R(I)+\frac{m^{2}}{4} \int \mathrm{d}^{4} x U[e(1), \cdots, e(\mathcal{N})] .
$$


The potential $U$, constructed in [24], will be presented below in a reformulation. Around flat backgrounds, the spectrum consists of one massless and $\mathcal{N}-1$ massive states. The vielbein description is elegant and was very convenient for showing the absence of the Boulware-Deser ghosts. For further work, see [25].

Here we are interested in interpreting (6.1) as a theory of $\mathcal{N}-1$ spin-2 fields in the presence of gravity. First one has to identify one of the vielbeins, say, $e_{\nu}^{b}(1)$, with the gravitational metric, $g_{\mu \nu}=e_{\mu}^{a}(1) \eta_{a b} e_{\nu}^{b}(1)$. Then, off shell, the $16(\mathcal{N}-1)$ components of the remaining vielbeins contain the $10(\mathcal{N}-1)$ degrees of freedom for describing $\mathcal{N}-1$ spin-2 fields as symmetric rank-2 tensors with kinetic terms consistent with general covariance. In addition, there are $6(\mathcal{N}-1)$ extra non-dynamical fields, as there are no leftover local Lorentz transformations to remove them. The latter, have to be eliminated through their equations of motion to isolate the spin-2 content of the theory. A difficulty that arises for $\mathcal{N}>2$ is in disentangling these non-dynamical components from the ones belonging to the spin-2 fields in kinetic terms [24].

In other words, from the remaining vielbeins, one can construct $\mathcal{N}-1$ rank-2 tensors $\theta_{\nu}^{\mu}(I)=e_{a}^{\mu}(1) e_{\nu}^{a}(I)$ of mixed symmetry. The potential is a function of the $\theta_{\nu}^{\mu}(I)$. It is difficult to extract from these the spin-2 fields that have kinetic terms, by solving the nondynamical equations. Even more difficult is doing so in a general covariant way.

This issue is addressed in the metric formulation of the multivielbein action (6.1) that was obtained, and argued to remain ghost-free, in [25]. In this setup, the non-dynamical fields are isolated from the spin-2 content in a generally covariant way without solving any equations of motion, making it appropriate for the considerations here. Then we work with the multi spin-2 action,

$$
\sum_{I=1}^{\mathcal{N}} \int \mathrm{d}^{4} x \sqrt{-g(I)} R(I)+\frac{m^{2}}{4} \int \mathrm{d}^{4} x T^{I_{1} \ldots I_{4}} U_{I_{1} \ldots I_{4}},
$$

where $T_{I_{1} I_{2} I_{3} I_{4}}$, totally symmetric in its indices, contains the free parameters of the theory and the multivielbein potential of [24] is reformulated to [25],

$$
\begin{aligned}
U_{I_{1} \cdots I_{4}}=\sqrt{-\operatorname{det} g(1)} \tilde{\epsilon}^{\mu_{1} \cdots \mu_{4}} \tilde{\epsilon}_{\nu_{1} \cdots \nu_{4}} & \\
& \times L_{\lambda_{1}}^{\nu_{1}}\left(I_{1}\right)\left[\sqrt{g^{-1}(1) g\left(I_{1}\right)}\right]_{\mu_{1}}^{\lambda_{1}} \cdots L_{\lambda_{4}}^{\nu_{4}}\left(I_{4}\right)\left[\sqrt{g^{-1}(1) g\left(I_{4}\right)}\right]_{\mu_{4}}^{\lambda_{4}} .
\end{aligned}
$$

In this expression the $L_{\lambda}^{\nu}(I)$ satisfy $g_{\mu \nu} L_{\rho}^{\mu}(I) L_{\sigma}^{\nu}(I)=g_{\rho \sigma}$ and $L_{\lambda}^{\nu}(1)=\delta_{\lambda}^{\nu}$. They carry the $6(\mathcal{N}-1)$ non-dynamical parameters as they do not enter the kinetic terms. They can be eliminated through their own equations of motion, but solving these equations is not necessary to identify the spin-2 content of the theory. In this form, the similarity to bimetric form as given in [19] is apparent.

The equations of motion obtained from (6.1) or (6.2) admit proportional background solutions,

$$
\bar{g}_{\mu \nu}(I)=c_{I}^{2} \bar{g}_{\mu \nu}, \quad \text { or } \quad \bar{e}_{\mu}^{a}(I)=c_{I} \bar{e}_{\mu}^{a},
$$


for $I=2, \cdots, \mathcal{N}$, and were we have denoted $g_{\mu \nu}(1) \equiv g_{\mu \nu}$ and $e_{\mu}^{a}(1) \equiv \bar{e}^{a}{ }_{\mu}$. The $c_{I}$ will be determined by the parameters of theory. In analogy with the bimetric case, one can introduce, for $I=2, \cdots, \mathcal{N}$,

$$
S(I) \equiv \sqrt{g^{-1} g(I)}, \quad M(I)=g S(I)-c_{I} g
$$

Since,

$$
g S(I)=[g S(I)]^{\mathrm{T}},
$$

the $M_{\mu \nu}(I)$ will be symmetric and represent the $\mathcal{N}-1$ massive spin-2 fields with vanishing expectation values in proportional backgrounds. These generalize the massive mode (4.14) of the bimetric theory in the picture that $g_{\mu \nu}$ is the gravitational metric. Note however that for generic coefficients $T_{I_{1} I_{2} I_{3} I_{4}}$ in (6.2), the actual mass eigenstates will be given by linear combinations of the $M_{\mu \nu}(I)$.

In terms of $g$ and $M(I)=g S(I)-c_{I} g$, the potential $\sqrt{-g} V(L(I) S(I))$ is a finite polynomial of its argument. The kinetic terms for the $M(I)$ will simply involve $\mathcal{N}-1$ copies of the corresponding terms in the bimetric case. The $L(I)$ are determined in terms of $g$ and the $M(I)$.

\section{Discussion}

The results have already been summarized in section 1 so here we only make some additional comments. The nonlinear massless and massive modes were introduced as an extension of the corresponding linear modes. It remains to be seen if they have a relevance directly at the nonlinear level. Although it is stated that the weak gravity limit is needed to approach GR solution in a generic sense, and that the non-vanishing VEV of the massive mode $M$ parameterizes deviations from GR, these effects have not yet been quantified. For example, note that the Bianchi constraints (2.10) are independent of $m_{g}$ and $m_{f}$ hence their nontrivial consequences will not be affected by the weak gravity limit.

Another feature of the bimetric theory is that in the $g-f$ formulation with $g$ as the gravitational metric, the kinetic energy of $f$ as well as its matter couplings affect gravity only through the potential $V\left(g^{-1} f\right)$. In the $g-M$ formulation there are direct couplings between $g$ and the kinetic term as well as matter interactions of $M$. However, after the $M$ equation of motion is imposed, the two sectors interact only through $V$ again. In this sense the couplings in spin-2 theories are maximally non-minimal.

\section{Acknowledgments}

We would like to thank Jonas Enander, Paolo Gondolo, Alexander Merle, Stefan Sjörs, Rachel Rosen and Bo Sundborg for useful discussions and comments. 


\section{A Curvature relations}

Here we provide a relation between Ricci tensors on a manifold endowed with two covariant derivatives (see, for example, [55]). This simplifies manipulation in bimetric theory.

General relations: Given any two derivative operators $\nabla$ and $\bar{\nabla}$, there exist a $(1,2)$ tensor field $C$ such that the actions on vectors $\omega_{\mu}$ are related by,

$$
\nabla_{\mu} \omega_{\nu}=\bar{\nabla}_{\mu} \omega_{\nu}-C_{\mu \nu}{ }^{\alpha} \omega_{\alpha}
$$

If $\nabla$ and $\bar{\nabla}$ are torsion free and compatible with metrics $g$ and $\bar{g}$, the tensor $C$ is given by,

$$
C_{\mu \nu}^{\alpha}=\frac{1}{2} g^{\alpha \beta}\left(\bar{\nabla}_{\mu} g_{\beta \nu}+\bar{\nabla}_{\nu} g_{\beta \mu}-\bar{\nabla}_{\beta} g_{\mu \nu}\right) .
$$

Defining the associated Riemann tensors by $\left[\nabla_{\mu}, \nabla_{\alpha}\right] \omega_{\nu}=-R_{\mu \alpha \nu}{ }^{\beta} \omega_{\beta}$, it is straightforward to derive a relation between the Ricci tensors $R_{\mu \nu}=R_{\mu \alpha \nu}{ }^{\alpha}$ as,

$$
R_{\mu \nu}(g)=R_{\mu \nu}(\bar{g})+2 \bar{\nabla}_{[\mu} C_{\alpha] \nu}{ }^{\alpha}-2 C_{\nu[\mu}{ }^{\beta} C_{\alpha] \beta}{ }^{\alpha} .
$$

Example: Linearizing General Relativity: Consider a metric $g$ as a perturbation around a background metric $\bar{g}, g_{\mu \nu}=\bar{g}_{\mu \nu}+\delta g_{\mu \nu}$. To linear order in $\delta g$, the curvature relation (A.3) gives,

$$
R_{\mu \nu}(g)=R_{\mu \nu}(\bar{g})+2 \bar{\nabla}_{[\mu} \delta \Gamma_{\alpha] \nu}{ }^{\alpha},
$$

where, with an obvious change of notation, $\delta \Gamma$ is given by the linear terms in (A.2),

$$
\delta \Gamma_{\mu \nu}^{\alpha}=\frac{1}{2} \bar{g}^{\alpha \beta}\left(\bar{\nabla}_{\mu} \delta g_{\beta \nu}+\bar{\nabla}_{\nu} \delta g_{\beta \mu}-\bar{\nabla}_{\beta} \delta g_{\mu \nu}\right) .
$$

This can be used to expand the Einstein equations, $R_{\mu \nu}-\frac{1}{2} g_{\mu \nu} R+\Lambda g_{\mu \nu}=\frac{1}{M_{P}^{2}} T_{\mu \nu}$, to linear order in $\delta g$. One gets the background and the fluctuation equations,

$$
\bar{R}_{\mu \nu}-\frac{1}{2} \bar{g}_{\mu \nu} \bar{R}+\Lambda \bar{g}_{\mu \nu}=\frac{1}{M_{P}^{2}} \bar{T}_{\mu \nu}, \quad \overline{\mathcal{E}}_{\mu \nu}^{\rho \sigma} \delta g_{\rho \sigma}+\Lambda \delta g_{\mu \nu}=\frac{1}{M_{P}^{2}} \delta T_{\mu \nu}
$$

where we have defined,

$$
\begin{aligned}
\overline{\mathcal{E}}_{\mu \nu}^{\rho \sigma} \delta g_{\rho \sigma}=-\frac{1}{2}\left[\delta_{\mu}^{\rho} \delta_{\nu}^{\sigma} \bar{\nabla}^{2}+\right. & \bar{g}^{\rho \sigma} \bar{\nabla}_{\mu} \bar{\nabla}_{\nu}-\delta_{\mu}^{\rho} \bar{\nabla}^{\sigma} \bar{\nabla}_{\nu}-\delta_{\nu}^{\rho} \bar{\nabla}^{\sigma} \bar{\nabla}_{\mu} \\
& \left.-\bar{g}_{\mu \nu} \bar{g}^{\rho \sigma} \bar{\nabla}^{2}+\bar{g}_{\mu \nu} \bar{\nabla}^{\rho} \bar{\nabla}^{\sigma}-\bar{g}_{\mu \nu} \bar{R}^{\rho \sigma}+\delta_{\mu}^{\rho} \delta_{\nu}^{\sigma} \bar{R}\right] \delta g_{\rho \sigma} .
\end{aligned}
$$

Using the background equation, the curvature contributions to $\overline{\mathcal{E}}_{\mu \nu}^{\rho \sigma}$ can be re-expressed in terms of $\bar{T}_{\mu \nu}$ and $\Lambda$.

\section{B Bimetric action in scaled variables}

The action (2.1) can be recast in a more symmetric form. Consider the rescalings,

$$
g_{\mu \nu}=\frac{m_{\mathrm{eff}}^{2}}{m_{g}^{2}} \tilde{g}_{\mu \nu}, \quad f_{\mu \nu}=\frac{m_{\mathrm{eff}}^{2}}{m_{f}^{2}} \tilde{f}_{\mu \nu}, \quad \beta_{n}=\left(\frac{m_{f}}{m_{g}}\right)^{n} \tilde{\beta}_{n}, \quad m^{4}=m_{g}^{4} \frac{\tilde{m}^{2}}{m_{\mathrm{eff}}^{2}} .
$$


In the new variables, the action is invariant under the interchanges $\tilde{g} \leftrightarrow \tilde{f}, \tilde{\beta}_{n} \leftrightarrow \tilde{\beta}_{4-n}$,

$$
S_{g f}=m_{\text {eff }}^{2} \int \mathrm{d}^{4} x\left[\sqrt{-\tilde{g}} R(\tilde{g})+\sqrt{-\tilde{f}} R(\tilde{f})-2 \tilde{m}^{2} \sqrt{-\tilde{g}} V\left(\sqrt{\tilde{g}^{-1} \tilde{f}}, \tilde{\beta}_{n}\right)\right] .
$$

The analysis of consistency of coupling the nonlinear massless mode to matter in section 4.3 is performed in terms of these variables.

\section{Details of the nonlinear $G-M^{G}$ action}

Below we work with the rescaled variables introduced above. For the sake of completeness, here we provide the details for writing the bimetric action in terms of the nonlinear massless and massive modes $G$ and $M^{G}$ (4.10), (4.15). To do this systematically, note that the expressions for $G$ and $M^{G}$ can be inverted to give,

$$
g_{\mu \nu}=G_{\mu \alpha}\left(\Phi^{-1}\right)^{\alpha}{ }_{\nu} \equiv \phi_{\mu \nu}, \quad f_{\mu \nu}=G_{\mu \alpha}\left(\tilde{\Phi}^{-1}\right)^{\alpha}{ }_{\nu} \equiv \tilde{\phi}_{\mu \nu} .
$$

Here $\Phi^{\alpha}{ }_{\nu}$ and $\tilde{\Phi}^{\alpha}{ }_{\nu}$ are functions of the matrix $S$ given by,

$$
\Phi_{\nu}^{\mu}=\delta_{\nu}^{\mu}+S^{\mu}{ }_{\alpha} S_{\nu}^{\alpha}, \quad \tilde{\Phi}^{\mu}{ }_{\nu}=\delta_{\nu}^{\mu}+\left(S^{-1}\right)^{\mu}{ }_{\alpha}\left(S^{-1}\right)^{\alpha}{ }_{\nu},
$$

and $S$ is related to the massive mode $M^{G}$ in a simple way,

$$
S=G^{-1} M^{G}+c \mathbb{1}
$$

These express $g$ and $f$ in terms of $G$ and $M^{G}$. We also define the inverse matrices,

$$
\phi^{\mu \alpha} \phi_{\alpha \nu}=\delta_{\nu}^{\mu}, \quad \tilde{\phi}^{\mu \alpha} \tilde{\phi}_{\alpha \nu}=\delta_{\nu}^{\mu}
$$

Using (A.3) the curvatures of $g$ and $f$ can be related to the curvature of $G$ and quantities that contain covariant derivatives only with respect to $G_{\mu \nu}$,

$$
R_{\mu \nu}(g)=R_{\mu \nu}(G)+2 \nabla_{[\mu} C_{\alpha] \nu}{ }^{\alpha}-2 C_{\nu[\mu}{ }^{\beta} C_{\alpha] \beta}{ }^{\alpha},
$$

where,

$$
C_{\mu \nu}^{\alpha}=\frac{1}{2} g^{\alpha \beta}\left(\nabla_{\mu} g_{\beta \nu}+\nabla_{\nu} g_{\beta \mu}-\nabla_{\beta} g_{\mu \nu}\right)
$$

Similarly we have that,

$$
R_{\mu \nu}(f)=R_{\mu \nu}(G)+2 \nabla_{[\mu} \widetilde{C}_{\alpha] \nu}{ }^{\alpha}-2 \widetilde{C}_{\nu[\mu}{ }^{\beta} \widetilde{C}_{\alpha] \beta}{ }^{\alpha},
$$

where,

$$
\widetilde{C}_{\mu \nu}^{\alpha}=\frac{1}{2} f^{\alpha \beta}\left(\nabla_{\mu} f_{\beta \nu}+\nabla_{\nu} f_{\beta \mu}-\nabla_{\beta} f_{\mu \nu}\right) .
$$

We further note that the volume densities can be expressed as,

$$
\sqrt{-\operatorname{det} g}=\sqrt{-\operatorname{det} G} \sqrt{\operatorname{det} \Phi^{-1}}, \quad \text { and } \quad \sqrt{-\operatorname{det} f}=\sqrt{-\operatorname{det} G} \sqrt{\operatorname{det} \tilde{\Phi}^{-1}}
$$


Using these relations we proceed to obtain the general structure of the nonlinear action.

Kinetic structure for $M^{G}$ : Using (C.1), (C.4), and (C.9), it is a straightforward but tedious algebraic exercise to show that (modulo a total derivative),

$$
\begin{aligned}
& \sqrt{-\operatorname{det} g} \phi^{\mu \nu}\left(2 \nabla_{[\mu} C_{\alpha] \nu}{ }^{\alpha}-2 C_{\nu[\mu}{ }^{\beta} C_{\alpha] \beta}{ }^{\alpha}\right) \\
& =\sqrt{-\operatorname{det} g} \frac{1}{4}\left(\phi^{\alpha \beta} \phi_{\kappa \lambda} \phi_{\rho \sigma}+2 \delta_{\lambda}^{\beta} \delta_{\sigma}^{\alpha} \phi_{\kappa \rho}-2 \delta_{\lambda}^{\alpha} \delta_{\rho}^{\beta} \phi_{\kappa \sigma}-\phi^{\alpha \beta} \phi_{\kappa \rho} \phi_{\lambda \sigma}\right) \nabla_{\alpha} \phi^{\kappa \rho} \nabla_{\beta} \phi^{\lambda \sigma} .
\end{aligned}
$$

A similar result is obtained for the corresponding term in the $f_{\mu \nu}$ sector by simply replacing $\Phi$ by $\tilde{\Phi}$ everywhere in the above (and $g$ by $f$ in the determinant prefactor). Next note that from (C.2) we have,

$$
\nabla_{\alpha} \Phi_{\nu}^{\mu}=\left(\delta_{\lambda}^{\mu} S_{\nu}^{\sigma}+\delta_{\nu}^{\sigma} S_{\lambda}^{\mu}\right) \nabla_{\alpha} S_{\sigma}^{\lambda}
$$

and also

$$
\nabla_{\alpha} \tilde{\Phi}_{\nu}^{\mu}=-\left(\left(S^{-1}\right)_{\lambda}^{\mu}\left(S^{-1}\right)_{\beta}^{\sigma}\left(S^{-1}\right)_{\nu}^{\beta}+\left(S^{-1}\right)_{\nu}^{\sigma}\left(S^{-1}\right)_{\beta}^{\mu}\left(S^{-1}\right)_{\lambda}^{\beta}\right) \nabla_{\alpha} S_{\sigma}^{\lambda} .
$$

These together with (C.10) and its corresponding expression for $f_{\mu \nu}$ give,

$$
\begin{aligned}
& \sqrt{-\operatorname{det} g} \phi^{\mu \nu}\left(2 \nabla_{[\mu} C_{\alpha] \nu}{ }^{\alpha}-2 C_{\nu[\mu}{ }^{\beta} C_{\alpha] \beta}{ }^{\alpha}\right)=\sqrt{-\operatorname{det} g} \mathcal{P}_{\alpha \beta}^{\lambda \sigma \kappa \rho} \nabla_{\lambda} S_{\sigma}^{\alpha}{ }_{\sigma} \nabla_{\kappa} S_{\rho}^{\beta}, \\
& \sqrt{-\operatorname{det} f} \tilde{\phi}^{\mu \nu}\left(2 \nabla_{[\mu} \widetilde{C}_{\alpha] \nu}{ }^{\alpha}-2 \widetilde{C}_{\nu[\mu}{ }^{\beta} \widetilde{C}_{\alpha] \beta}{ }^{\alpha}\right)=\sqrt{-\operatorname{det} f} \widetilde{\mathcal{P}}_{\alpha \beta}^{\lambda \sigma \kappa \rho} \nabla_{\lambda} S^{\alpha}{ }_{\sigma}{ }^{\alpha} \nabla_{\kappa} S_{\rho}^{\beta},
\end{aligned}
$$

in terms of the polarization tensors $\mathcal{P}$ and $\widetilde{\mathcal{P}}$. We refrain from writing out the full expressions for these tensors here and simply note that they can be straightforwardly deduced from (C.10), (C.11) and (C.12). Since,

$$
\nabla_{\kappa} S_{\rho}^{\beta}=G^{\beta \lambda} \nabla_{\kappa} M_{\lambda \rho}^{G},
$$

equations (C.13) and (C.14) provide the kinetic term for massive field $M^{G}$ with only $G$ covariant derivatives.

Kinetic structure for $G$ : From (C.5) and (C.7) combined with (C.1) we find the corresponding relations for the Ricci scalars,

$$
\begin{aligned}
& R(g)=R(G)+S_{\alpha}^{\mu} S_{\beta}^{\alpha} G^{\beta \nu} R_{\mu \nu}(G)+\ldots \\
& R(f)=R(G)+\left(S^{-1}\right)_{\alpha}^{\mu}\left(S^{-1}\right)^{\alpha}{ }_{\beta} G^{\beta \nu} R_{\mu \nu}(G)+\ldots
\end{aligned}
$$

where the dots represents the kinetic terms for $M^{G}$ discussed above. Hence, the kinetic structure for $G$ is given by the usual Einstein-Hilbert term plus non-minimal coupling of the Ricci tensor to $S$ in the $g_{\mu \nu}$ sector and to $S^{-1}$ in the $f_{\mu \nu}$ sector. Apart from this we also take into consideration the volume densities given by (C.9). Thus, the full kinetic structure for $G$ is given by (omitting the overall factor of $\sqrt{-\operatorname{det} G}$ ),

$$
\begin{aligned}
\left(\sqrt{\operatorname{det} \Phi^{-1}}+\sqrt{\operatorname{det} \tilde{\Phi}^{-1}}\right) R(G) & +\sqrt{\operatorname{det} \Phi^{-1}} S^{\mu}{ }_{\alpha} S_{\beta}^{\alpha} G^{\beta \nu} R_{\mu \nu}(G) \\
& +\sqrt{\operatorname{det} \tilde{\Phi}^{-1}}\left(S^{-1}\right)_{\alpha}^{\mu}\left(S^{-1}\right)^{\alpha}{ }_{\beta} G^{\beta \nu} R_{\mu \nu}(G) .
\end{aligned}
$$


This relation together with (C.13) and (C.14) completely determine the kinetic terms.

Full nonlinear $G-M^{G}$ action: Using (C.3), the interaction potential is easily expressed in terms of $M^{G}$ as in (5.2),

$$
\sqrt{-\operatorname{det} g} V\left(S, \beta_{n}\right)=\sqrt{-\operatorname{det} G}\left(1+S^{2}\right)^{-1 / 2} V\left(M^{G}, \alpha_{n}^{c}\right) .
$$

Collecting all the results, we can now write the Lagrangian in (B.2) in terms of $G$ and $M^{G}$ as,

$$
\begin{aligned}
\mathcal{L}\left(G, M^{G}\right)= & \left(\operatorname{det}\left(1+S^{2}\right)^{-1 / 2}+\operatorname{det}\left(1+S^{-2}\right)^{-1 / 2}\right) R(G) \\
& +\left(\operatorname{det}\left(1+S^{2}\right)^{-1 / 2} \mathcal{P}_{\alpha \beta}^{\lambda \sigma \kappa \rho}+\operatorname{det}\left(1+S^{-2}\right)^{-1 / 2} \widetilde{\mathcal{P}}_{\alpha \beta}^{\lambda \sigma \kappa \rho}\right) \nabla_{\lambda} S_{\sigma}^{\alpha} \nabla_{\kappa} S_{\rho}^{\beta} \\
& +\operatorname{det}\left(1+S^{2}\right)^{-1 / 2} S^{\mu}{ }_{\alpha} S^{\alpha}{ }_{\beta} G^{\beta \nu} R_{\mu \nu}(G) \\
& +\operatorname{det}\left(1+S^{-2}\right)^{-1 / 2}\left(S^{-1}\right)^{\mu}{ }_{\alpha}\left(S^{-1}\right)^{\alpha}{ }_{\beta} G^{\beta \nu} R_{\mu \nu}(G) \\
& -2 m^{2} \operatorname{det}\left(1+S^{2}\right)^{-1 / 2} V\left(M^{G}, \alpha_{n}^{c}\right)
\end{aligned}
$$

such that the full action is given by

$$
S_{G M}=m_{\text {eff }}^{2} \int \mathrm{d}^{4} x \sqrt{-\operatorname{det} G} \mathcal{L}\left(G, M^{G}\right) .
$$

\section{References}

[1] N. Rosen, Phys. Rev. 57 (1940) 150.

[2] C. J. Isham, A. Salam and J. A. Strathdee, Phys. Rev. D 3 (1971) 867.

[3] C. Aragone and S. Deser, Nuovo Cim. A 3 (1971) 709.

[4] N. Rosen, In *Erice 1975, Proceedings, Topics In Theoretical and Experimental Gravitation Physics*, New York 1977, 273-294

[5] A. H. Chamseddine, A. Salam and J. A. Strathdee, Nucl. Phys. B 136 (1978) 248.

[6] C. Aragone and S. Deser, Nuovo Cim. B 57 (1980) 33.

[7] I. L. Buchbinder, V. A. Krykhtin and V. D. Pershin, Phys. Lett. B 466 (1999) 216 [hep-th/9908028].

[8] I. L. Buchbinder, D. M. Gitman, V. A. Krykhtin and V. D. Pershin, Nucl. Phys. B 584 (2000) 615 [hep-th/9910188].

[9] N. Boulanger, T. Damour, L. Gualtieri and M. Henneaux, Nucl. Phys. B 597 (2001) 127 [hep-th/0007220].

[10] T. Damour and I. I. Kogan, Phys. Rev. D 66 (2002) 104024 [hep-th/0206042].

[11] T. Damour, I. I. Kogan and A. Papazoglou, Phys. Rev. D 66 (2002) 104025 [hep-th/0206044].

[12] N. Arkani-Hamed, H. Georgi and M. D. Schwartz, Annals Phys. 305 (2003) 96 [hep-th/0210184]. 
[13] D. Blas, C. Deffayet and J. Garriga, Phys. Rev. D 76 (2007) 104036 [arXiv:0705.1982 [hep-th]].

[14] M. Banados, A. Gomberoff, D. C. Rodrigues and C. Skordis, Phys. Rev. D 79 (2009) 063515 [arXiv:0811.1270 [gr-qc]].

[15] M. Milgrom, Phys. Rev. D 80 (2009) 123536 [arXiv:0912.0790 [gr-qc]].

[16] D. G. Boulware and S. Deser, Phys. Rev. D 6 (1972) 3368.

[17] S. F. Hassan and R. A. Rosen, JHEP 1202 (2012) 126 [arXiv:1109.3515 [hep-th]].

[18] S. F. Hassan and R. A. Rosen, JHEP 1204 (2012) 123 [arXiv:1111.2070 [hep-th]].

[19] S. F. Hassan and R. A. Rosen, JHEP 1107 (2011) 009 [arXiv:1103.6055 [hep-th]].

[20] S. F. Hassan and R. A. Rosen, Phys. Rev. Lett. 108 (2012) 041101 [arXiv:1106.3344 [hep-th]].

[21] S. F. Hassan, R. A. Rosen and A. Schmidt-May, JHEP 1202 (2012) 026 [arXiv:1109.3230 [hep-th]].

[22] C. de Rham and G. Gabadadze, Phys. Rev. D 82 (2010) 044020 [arXiv:1007.0443 [hep-th]].

[23] C. de Rham, G. Gabadadze and A. J. Tolley, Phys. Rev. Lett. 106 (2011) 231101 [arXiv:1011.1232 [hep-th]].

[24] K. Hinterbichler and R. A. Rosen, JHEP 1207 (2012) 047 [arXiv:1203.5783 [hep-th]].

[25] S. F. Hassan, A. Schmidt-May and M. von Strauss, arXiv:1204.5202 [hep-th].

[26] M. S. Volkov, JHEP 1201 (2012) 035 [arXiv:1110.6153 [hep-th]].

[27] M. S. Volkov, arXiv:1202.6682 [hep-th].

[28] M. von Strauss, A. Schmidt-May, J. Enander, E. Mortsell and S. F. Hassan, JCAP 1203 (2012) 042 [arXiv:1111.1655 [gr-qc]].

[29] D. Comelli, M. Crisostomi, F. Nesti and L. Pilo, Phys. Rev. D 85 (2012) 024044 [arXiv:1110.4967 [hep-th]].

[30] D. Comelli, M. Crisostomi, F. Nesti and L. Pilo, JHEP 1203 (2012) 067 [Erratum-ibid. 1206 (2012) 020] [arXiv:1111.1983 [hep-th]].

[31] V. Baccetti, P. Martin-Moruno and M. Visser, arXiv:1206.4720 [gr-qc].

[32] C. Deffayet and T. Jacobson, Class. Quant. Grav. 29 (2012) 065009 [arXiv:1107.4978 [gr-qc]].

[33] M. Fierz and W. Pauli, Proc. Roy. Soc. Lond. A 173 (1939) 211.

[34] W. Pauli and M. Fierz, Helv. Phys. Acta 12 (1939) 297.

[35] P. Creminelli, A. Nicolis, M. Papucci and E. Trincherini, JHEP 0509 (2005) 003 [hep-th/0505147].

[36] R. L. Arnowitt, S. Deser and C. W. Misner, [gr-qc/0405109].

[37] M. Mirbabayi, arXiv:1112.1435 [hep-th].

[38] C. de Rham and S. Renaux-Petel, arXiv:1206.3482 [hep-th].

[39] J. Kluson, JHEP 1201 (2012) 013 [arXiv:1109.3052 [hep-th]].

[40] A. H. Chamseddine and V. Mukhanov, JHEP 1108 (2011) 091 [arXiv:1106.5868 [hep-th]].

[41] S. F. Hassan, A. Schmidt-May and M. von Strauss, arXiv:1203.5283 [hep-th]. 
[42] J. Kluson, arXiv:1204.2957 [hep-th].

[43] V. Baccetti, P. Martin-Moruno and M. Visser, arXiv:1205.2158 [gr-qc].

[44] V. Baccetti, P. Martin-Moruno and M. Visser, arXiv:1206.3814 [gr-qc].

[45] N. Khosravi, N. Rahmanpour, H. R. Sepangi and S. Shahidi, Phys. Rev. D 85 (2012) 024049 [arXiv:1111.5346 [hep-th]].

[46] N. Khosravi, H. R. Sepangi and S. Shahidi, Phys. Rev. D 86 (2012) 043517 [arXiv:1202.2767 [gr-qc]].

[47] E. N. Saridakis, arXiv:1207.1800 [gr-qc].

[48] Y. -F. Cai, C. Gao and E. N. Saridakis, JCAP 1210 (2012) 048 [arXiv:1207.3786 [astro-ph.CO]].

[49] A. E. Gumrukcuoglu, C. Lin and S. Mukohyama, JCAP 1203 (2012) 006 [arXiv:1111.4107 [hep-th]].

[50] M. Crisostomi, D. Comelli and L. Pilo, JHEP 1206 (2012) 085 [arXiv:1202.1986 [hep-th]].

[51] M. Berg, I. Buchberger, J. Enander, E. Mortsell and S. Sjors, arXiv:1206.3496 [gr-qc].

[52] M. F. Paulos and A. J. Tolley, arXiv:1203.4268 [hep-th].

[53] T. Ortin, Cambridge Unversity, Cambridge University Press, 2004

[54] B. Grinstein, C. W. Murphy, D. Pirtskhalava and P. Uttayarat, arXiv:1203.2183 [hep-ph].

[55] R. M. Wald, Chicago, Usa: Univ. Pr. ( 1984) 491p. 